\title{
Approximating the physical inner product of loop quantum cosmology
}

\author{
Benjamin Bahr ${ }^{1}$ and Thomas Thiemann ${ }^{2}$ \\ ${ }^{1}$ MPI für Gravitationsphysik, Albert-Eintstein Institut, Am Mühlenberg 1, 14467 Golm, Germany \\ ${ }^{2}$ Permineter Institute for Theoretical Physics, 31 Caroline St. N., Waterloo, Ontario N2L 2Y5, \\ Canada
}

Received 22 November 2006, in final form 14 February 2007

Published 28 March 2007

Online at stacks.iop.org/CQG/24/2109

\begin{abstract}
In this paper, we investigate the possibility of approximating the physical inner product of constrained quantum theories. In particular, we calculate the physical inner product of a simple cosmological model in two ways: firstly, we compute it analytically via a trick; secondly, we use the complexifier coherent states to approximate the physical inner product defined by the master constraint of the system. We find that the approximation is able to recover the analytic solution of the problem, which consolidates hopes that coherent states will help to approximate solutions of more complicated theories, like loop quantum gravity.
\end{abstract}

PACS number: $04.60 . \mathrm{Pp}$

\section{Introduction}

Loop quantum gravity is an attempt to quantize general relativity (GR) to obtain a quantum theory of gravity (see [22] and references therein). In order to do so, one first has to rewrite GR as a Hamiltonian theory, which leads to a constrained system. The question of how to quantize a Hamiltonian theory with constraints has existed for a long time $[6,9,13]$. The technical issues are, in fact, not too complicated. The constraint functions $C_{i}$, which are phasespace functions in the classical theory, become operators $\hat{C}_{i}$ on an auxiliary, or kinematical Hilbert space $\mathcal{H}_{\text {kin. }}$. The classical issue of solving the constraints is, on the quantum side, transformed into the task of computing the common zero of the constraint operators $\hat{C}_{i} \psi=0$. Unfortunately, since the point zero is generically in the continuous parts of the spectra of the $\hat{C}_{i}$, the space of solutions to the constraints will in general be no subspace of the Hilbert space. Rather, the set of solutions will contain distributions which will not be normalizable in the inner product on $\mathcal{H}_{\text {kin. }}$. So, even when the space of solutions of $\hat{C}_{i} \psi=0$ is known, it has no structure of a Hilbert space yet. In fact, from various examples it is known that the choice of a 'physical' inner product is a quite nontrivial task, and the resulting theory will crucially 
depend on this choice. Generically, a lot of choices are possible which result in trivial theories, or nontrivial theories that in no way resemble the features of the classical theories one started with.

In loop quantum gravity this issue is even more complicated, since the algebra of constraints is tremendously difficult. Although it is possible to define the Hamiltonian constraints [16-18] (which then still contain a lot of freedom), the diffeomorphism constraints cannot be implemented as operators on $\mathcal{H}_{\text {kin }}$ [22]. Furthermore, there are arguments that even if such operators could be constructed, they could not be self-adjoint, which makes spectral analysis quite difficult (see, e.g. remarks in [19, 22]). Nevertheless, a diffeomorphism invariant Hilbert space $\mathcal{H}_{\text {diff }}$ could be defined, which contained vectors invariant under the exponentiated diffeomorphism operators [3]. But since the Hamiltonian constraints do not even weakly commute with the diffeomorphism constraints, there was no way of defining the Hamiltonian constraints on $\mathcal{H}_{\text {diff }}$, so the search for the solutions to the constraints could not continue.

In 2003, a solution to this set of problems was suggested [19]. It is mainly motivated by the observation that a classical constrained system with an arbitrary number of constraints $C_{i}$ can be replaced by an equivalent system with only one constraint, the so-called master constraint $M$. Solving for all the $C_{i}$ simultaneously or solving for $M$ then leads to identical solutions. In the corresponding quantum theories the constraint operators $\hat{C}_{i}$ can be replaced by the master constraint operator $\hat{M}$. Unfortunately, while on the classical side these two sets of constraints lead to equivalent classical theories, this equivalence is not that obvious in quantum theory. In generic situations, there are a lot more solutions to $\hat{M} \psi=0$ than to $\hat{C}_{i} \psi=0$ for all $i$. On the other hand, in generic models, not even all of the solutions to the constraint, whenever one was able to compute them, had non-vanishing physical norm, electromagnetism being the first example. In many tested models, many of the formal solutions of $\hat{M}=0$ also turned out to have zero norm in the physical inner product [8]. This reinforced the hope that the master constraint could be used to define the physical inner product and lead to sensible results: there was the possibility that the additional solutions were all spurious, i.e. had all zero physical norm.

Still, even with the master constraint, the task of computing the physical inner product is, for the case of loop quantum gravity, technically very hard [20]. The operator $\hat{M}$ is quite complicated and hence a spectral analysis is presumably not possible analytically. On the other hand, this is nothing to be seriously concerned about, since knowledge of the physical inner product would be equivalent to a complete solution of the theory, i.e. a quantum theory which, in its semiclassical sector, is believed to contain classical general relativity. But even of classical GR one does not know all solutions (in fact, one does not even know how many solutions one does not know). So solving the complete theory is not something we can expect at all. On the other hand, this is also not something that is necessary in order to do physics. The situation is similar to the case of condensed matter physics, where the Hamiltonian operator for a crystal with roughly $10^{23}$ particles can be written down, but not one solution is known. And this is not necessary in order to do physics, since no exact solutions are needed to make predictions about conductivity, magnetization or the shear module of a crystal.

So what one needs in order to extract physics from the equations of LQG are approximation schemes for the physical inner product defined by $\hat{M}$. In this paper, we will investigate a simple cosmological model with only one constraint $\hat{C}$, and try to approximate the physical inner product defined by the corresponding master constraint $\hat{M}$ with the help of complexifier coherent states. These states were introduced by Hall [10-12] and investigated in [21, 23-25]. They have properties that make them useful tools for semiclassical analysis; in particular, they will allow for an approximation for the physical inner product of our toy model. 
There are, with some tricks, other possibilities of calculating the physical inner product in this model analytically [14], without the need to employ the master constraint $\hat{M}$, and we will see that the approximation provided by the coherent states in fact works. Furthermore, the solutions to the master constraint $\hat{M}$ that are not solutions to the constraint $\hat{C}$ turn out not to be in the physical Hilbert space, as one hopes.

The constraint operator we investigate was introduced in [2]. Recently, modifications to this constraint operator have been proposed [5]. We will briefly comment on the implications this change would have for our results.

\section{Preliminaries}

\subsection{The master constraint}

Consider a phase space $\mathcal{M}$ and a set of first class constraints $C_{i}$. This means that the Poisson brackets between two constraint functions $\left\{C_{i}, C_{j}\right\}$ vanish at the phase-space points where all the constraints $C_{i}$ vanish. Then the space of all points $m \in \mathcal{M}$ with $C_{i}(m)=0$ for all $i$ is called the reduced phase space. The Hamiltonian vector fields of the constraints $X_{C_{i}}$ generate flows that leave this reduced phase space invariant, and the orbit space is called the physical phase space. All phase-space functions weakly commuting with the constraints, called weak Dirac observables, can then be made into functions on the physical phase space, and will serve as observables.

Instead of solving all the (possibly infinitely many) equations $C_{i}(m)=0$, one can also define the so-called master constraint $[19,20]$

$$
M:=\sum_{i} C_{i} K_{i j} C_{i}
$$

Here $K_{i j}$ is a symmetric, positive definite matrix in the case of $i$ being a discrete set. Otherwise, $K_{i j}$ has to be a positive definite operator kernel and the summation over $i$ turns into an integration. It is straightforward to see that

$$
M(m)=0 \quad \Leftrightarrow \quad C_{i}(m)=0 \quad \text { for all } i .
$$

Furthermore, for any phase-space function $f$ weakly commuting with the constraints:

$$
\{\{M, f\}, f\} \approx 0, \quad \Leftrightarrow \quad\left\{C_{i}, f\right\} \approx 0 \quad \text { for all } i .
$$

So $M$ enables us to derive the complete set of observables on the physical pase space. This means that the physical phase space itself can be constructed from knowledge of $M$, so one does not lose any information if one goes over to $M$ from the $C_{i}$. The final classical systems defined by both are in fact equivalent. This is in fact independent of the actual choice of $K_{i j}$, so there are a priori many possible master constraints. We can choose the one that is most useful, makes the sum (2.2) converge and is the most convenient to compute. This freedom is quite useful in quantized theories [8].

\subsection{Quantizing constrained systems}

Consider a Hilbert space $\mathcal{H}_{\text {kin }}$ and a set of constraint operators $\hat{C}_{i}$. Let furthermore $\mathscr{D}_{\text {kin }} \subset \mathcal{H}_{\text {kin }}$ be a dense subspace which is invariant under the action of the $\hat{C}_{i}$ and their adjoints $\hat{C}_{i}^{\dagger}$. A solution to the constraints is a linear form

$$
l: \mathscr{D}_{\text {kin }} \rightarrow \mathbb{C}
$$

with

$$
l \circ \hat{C}_{i}^{\dagger}=0 \quad \text { for all } \quad i
$$


The space of all such linear forms $\mathscr{D}_{\text {phys }}^{*}$ is a subspace of the algebraic dual of $\mathscr{D}_{\text {kin }}$ :

$$
\mathscr{D}_{\text {phys }}^{*} \subset \mathscr{D}_{\text {kin }}^{*} \text {. }
$$

In general, $\mathscr{D}_{\text {phys }}^{*} \cap \mathcal{H}_{\text {kin }}=\{0\}$, so the solutions to the constraints will not be normalizable in the inner product on $\mathcal{H}_{\text {kin }}$. Furthermore, not only can $\mathscr{D}_{\text {phys }}^{*}$ not inherit the inner product from $\mathcal{H}_{\text {kin }}$, experience also shows that not all elements in $\mathscr{D}_{\text {phys }}^{*}$ are acceptable as solutions for generic models. Generally, $\mathscr{D}_{\text {phys }}^{*}$ is 'too big' to admit a positive definite inner product.

The task is now to find a Hilbert space $\mathcal{H}_{\text {phys }}$ that is a subspace of $\mathscr{D}_{\text {phys }}^{*}$. In general, there will be a lot of them, and the issue of finding the 'right' one is a nontrivial question. One necessary condition for this Hilbert space would for instance be that it supports the quantizations of observables weakly commuting with the constraints $C_{i}$ as self-adjoint operators.

The idea is now to replace the set of constraint operators $\hat{C}_{i}$ by the quantization of (2.1), the master constraint operator

$$
\hat{M}:=\sum_{i} \hat{C}_{i} K_{i j} \hat{C}_{i}^{\dagger}
$$

Here the freedom to choose $K_{i j}$ has in fact to be exploited in order to arrive at a welldefined operator.

One can immediately see that $\mathscr{D}_{\text {kin }}$ is left invariant by $\hat{M}$, and for any linear form $l \in \mathscr{D}_{\text {kin }}^{*}$ :

$$
l \circ \hat{C}_{i}=0 \quad \text { for all } i \quad \Rightarrow \quad l \circ \hat{M}=0 .
$$

If 0 is only contained in the discrete (point) spectrum of all the $\hat{C}_{i}$, then (2.8) is an equivalence relation. But if 0 is in the continuous part of the spectrum, then the converse of then (2.8) need not be true. So the space of solutions of $\hat{M}$ is potentially larger than the space of solutions to the constraints $\hat{C}_{i}$. On the other hand, $\hat{M}$ provides a natural way to define the physical Hilbert space. In all models checked so far, this Hilbert space turned out to contain no solutions of $\hat{M}$ that were no solutions to the $\hat{C}_{i}$. Although there are no hard proofs available at the moment, there are high hopes that this is the case generally.

\subsection{The physical Hilbert space and rigging maps}

Since $\hat{M}$ is formally symmetric and non-negative by construction, one can always extend it to a self-adjoint operator. Of course, this has to be checked from case to case, and we assume it to be true here. Then $\hat{M}$ is also positive and hence its spectrum is a subset of $[0, \infty)$. We furthermore assume that 0 is in fact in the spectrum of $\hat{M}$. Then, for each $\lambda \in \operatorname{spec}(\hat{M})$ there is a Hilbert space $\mathcal{H}_{\lambda}$, equipped with inner product $\langle\cdot \mid \cdot\rangle_{\lambda}$. Furthermore there is a measure $\mu$ on $\operatorname{spec}(\hat{M})$ such that each vector of $\mathcal{H}_{\text {kin }}$ can be decomposed into

$$
\psi=\int_{\operatorname{spec}(\hat{M})} \mathrm{d} \mu(\lambda) \psi(\lambda)
$$

and the inner product in $\mathcal{H}_{\text {kin }}$ via

$$
\langle\psi \mid \phi\rangle=\int_{\operatorname{spec}(\hat{M})} \mathrm{d} \mu(\lambda)\langle\psi(\lambda) \mid \phi(\lambda)\rangle_{\lambda} .
$$

These facts can be abbreviated by

$$
\mathcal{H}_{\text {kin }}=\int_{\operatorname{spec}(\hat{M})}^{\oplus} \mathrm{d} \mu(\lambda) \mathcal{H}_{\lambda}
$$


which is called the direct integral decomposition of $\mathcal{H}_{\text {kin }}$. We neglect all discussions about the mathematical subtleties of this construction and just state the fact that the Hilbert space $\mathcal{H}_{\lambda}$ for $\lambda=0$ shall serve as a physical Hilbert space:

$$
\mathcal{H}_{\text {phys }}:=\mathcal{H}_{0}, \quad\langle\cdot \mid \cdot\rangle_{\text {phys }}:=\langle\cdot \mid \cdot\rangle_{0} .
$$

This procedure can, in principle, be applied to every system with constraints. In particular, in the case of 0 being in the discrete part of the spectrum only, this is just the definition of zero eigenspace of $\hat{M}$. In the case of a mixed spectrum of $\hat{M}$, one has to decompose $\mathcal{H}_{\text {kin }}$ into a 'discrete' and 'continuous' part and perform this analysis for each part separately:

$\mathcal{H}_{\text {kin }}=\mathcal{H}_{\text {kin }}^{p p} \oplus \mathcal{H}_{\text {kin }}^{a c}=\int_{\operatorname{spec}_{p p}(\hat{M})}^{\oplus} \mathrm{d} \mu_{p p}(\lambda) \mathcal{H}_{\lambda}^{p p} \oplus \int_{\operatorname{spec}_{a c}(\hat{M})}^{\oplus} \mathrm{d} \mu_{a c}(\lambda) \mathcal{H}_{\lambda}^{a c}$.

Here 'pp' and 'ac' denote the pure-point- and the absolutely continuous part of the spectrum of $\hat{M}$. In the end, the physical solutions from each separate part constitute the whole physical Hilbert space, in particular

$$
\mathcal{H}_{\text {phys }}=\mathcal{H}_{0}^{p p} \oplus \mathcal{H}_{0}^{a c} .
$$

Note that $\mathcal{H}_{0}^{p p} \subset \mathcal{H}_{\text {kin }}$ consists of the zero eigenvectors of $\hat{M}$. For both cases 'pp' and 'ac' the Hilbert space $\mathcal{H}_{0}$ is a subspace of $\mathscr{D}_{\text {phys }}^{*}$ in the following sense: in the direct integral decomposition $\psi \in \mathcal{H}_{\text {kin }}$ corresponds to a map $\lambda \mapsto \psi(\lambda)$ with $\psi(\lambda) \in \mathcal{H}_{\lambda}$. Denote the evaluation of this map at $\lambda=0$ as $\psi(0)$. Then define for each $\psi \in \mathcal{H}_{\text {kin }}$ the following linear functional:

$$
\eta[\psi]: \phi \longmapsto\langle\psi(0) \mid \phi(0)\rangle_{0} .
$$

So $\eta$ provides a 'projection' of $\mathcal{H}_{\text {kin }}$ in $\mathcal{H}_{0}$ (which is a proper projection for the pure-point case). On the other hand, since the decomposition of $\hat{M} \phi$ is given by the map $\lambda \mapsto \lambda \phi(\lambda)$, it is immediately clear that $\eta$ as defined in (2.15) maps $\psi$ into a solution of the constraint $\hat{M}$, i.e. $\eta[\psi] \circ \hat{M}=0$. In the literature one also finds for $\eta$ the name rigging map.

Although conceptually quite clear, the decomposition (2.11) is in general nontrivial, as the spectral analysis for an arbitrary self-adjoint operator can be highly complicated. This is also the fact for the master constraint operator of LQG. Although the operator has been constructed explicitly [20], its complete spectral analysis is currently out of reach.

\subsection{The operator $\delta(\hat{M})$}

We would now like to find a way of approximating the solutions of $\hat{M}$, i.e. the linear functionals (2.15) for all $\psi \in \mathcal{H}_{\text {kin. }}$. For this we observe

$$
\begin{aligned}
\eta[\psi] \phi & =\langle\psi(0) \mid \phi(0)\rangle_{0} \\
& =\int_{\operatorname{spec}(\hat{M})} \mathrm{d} \mu(\lambda)\langle\psi(\lambda)|\delta(\lambda)| \phi(\lambda)\rangle_{\lambda} \\
& =\langle\psi|\delta(\hat{M})| \phi\rangle,
\end{aligned}
$$

where $\delta(\hat{M})$ depends on $\mathrm{d} \mu(\lambda)$. The 'operator' $\delta(\hat{M})$ is, of course, no operator but a quadratic form which will not exist for all $\psi, \phi \in \mathcal{H}_{\text {kin }}$, but only for those vectors, whose decomposition into $\lambda \mapsto \psi(\lambda)$ is regular at $\lambda=0$.

Equation (2.16) now enables us to express the linear form $\eta[\psi]$ without directly computing the direct integral decomposition. All we need is the construction of a $\delta$-function for the measure $\mu$ on the spectrum of $\hat{M}$. For this, the knowledge of the measure $\mu$ is, of course, sufficient, but not necessary. All one needs to know is the rate at which the measure 'diverges' or 'goes to zero' at $\lambda=0$. 
If 0 is in the discrete part of the spectrum, the measure $\mu$ is a Dirac measure there, and the delta function is just the usual Kronecker delta. In this case the integral (2.11) is just a sum over $\lambda=0$ and hence $\delta(\hat{M})$ is a proper projection operator which maps $\mathcal{H}_{\text {kin }}$ onto its subspace $\mathcal{H}_{0}^{p p}$.

If 0 is in the continuous part of the spectrum, the measure $\mu$ can behave very singularly at $\lambda=0$. This is supported by the following observation: consider the direct integral decomposition of a self-adjoint operator $\hat{A}$, whose spectrum is the real line, and whose spectral measure $\mu_{\hat{A}}$ is the Lebesgue measure $\mathrm{d} \lambda$. Then the operator $\hat{A}^{2}$ has $[0, \infty)$ as spectrum, and its measure $\mu_{\hat{A}^{2}}$ is then $\frac{1}{2 \sqrt{\lambda}} \mathrm{d} \lambda$, which diverges as $\lambda$ tends to zero. So by taking powers of operators, one changes the behaviour of the spectral measure at $\lambda=0$.

So, an educated guess for a delta sequence is the functions

$$
\lambda \longmapsto t^{-\alpha} \mathrm{e}^{-\frac{\lambda^{2}}{t}}
$$

If the parameter $\alpha \in \mathbb{R}$ is chosen correctly, so as to match the degree of divergence of the measure $\mu$ at $\lambda=0$, then this diverges to a multiple of the $\delta$-distribution at $\lambda=0$, as $t$ tends to 0 . Determining $\alpha$ can be nontrivial. On the other hand, there is a different way of calculating the physical inner product without the need to know $\alpha$ explicitly: choose a vector $\psi \in \mathcal{H}_{\text {kin }}$ such that in its direct integral decomposition one has

$$
0<\|\psi(0)\|_{0}<\infty \text {. }
$$

Then one has

$$
\begin{aligned}
\lim _{t \rightarrow 0} \frac{\mathrm{e}^{-\frac{\hat{M}^{2}}{t}}}{\left\langle\psi\left|\mathrm{e}^{-\frac{\hat{M}^{2}}{t}}\right| \psi\right\rangle} & =\lim _{t \rightarrow 0} \frac{t^{-\alpha} \mathrm{e}^{-\frac{\hat{M}^{2}}{t}}}{t^{-\alpha}\left\langle\psi\left|\mathrm{e}^{-\frac{\hat{M}^{2}}{t}}\right| \psi\right\rangle}=\lim _{t \rightarrow 0} \frac{t^{-\alpha} \mathrm{e}^{-\frac{\hat{M}^{2}}{t}}}{t^{-\alpha} \int \mathrm{d} \mu(\lambda)\left\langle\psi(\lambda)\left|\mathrm{e}^{-\frac{\lambda^{2}}{t}}\right| \psi(\lambda)\right\rangle_{\lambda}} \\
& =\frac{\lim _{t \rightarrow 0} t^{-\alpha} \mathrm{e}^{-\frac{\hat{M}^{2}}{t}}}{\lim _{s \rightarrow 0} s^{-\alpha} \int \mathrm{d} \mu(\lambda)\left\langle\psi(\lambda)\left|\mathrm{e}^{-\frac{\lambda^{2}}{s}}\right| \psi(\lambda)\right\rangle_{\lambda}} \\
& =\frac{1}{\|\psi(0)\|_{0}} \delta(\hat{M}) .
\end{aligned}
$$

Here, the equalities have to be understood in the sense of quadratic forms. This shows that, by employing a 'reference vector' $\psi$ one can avoid the need to explicitly calculate $\alpha$. The resulting physical inner product is then rescaled such that $\eta[\psi]$ has unit norm in the physical inner product. One has now shifted the problem of finding $\alpha$ to finding a reference vector that satisfies (2.18). But since the set of vectors satisfying (2.18) is dense in $\mathcal{H}_{\text {kin }}$, one would think that finding such a vector is not too complicated.

\section{The model}

As a test of full LQG one can focus attention on symmetry truncations of LQG, in the hope to get technical insight into soluable situations which are still valid in the full theory. A particular class of truncations modelling cosmological situations, called loop quantum cosmology (LQC), has received a lot of interest recently [2]. We will apply the approximation scheme we have in mind to the easiest LQC model possible: that of a spatially homogenous and isotropic universe without any matter fields. For the case of a nonzero cosmological constant, the physical Hilbert space has been constructed in [14]. The case for vanishing cosmological constant is different, since then the zero is not in the discrete part of the spectrum any longer. It is this case that we will investigate in this paper.

The metric the model describes is

$$
\mathrm{d} s^{2}=-\mathrm{d} t^{2}+a(t)^{2}\left(\mathrm{~d} x^{2}+\mathrm{d} y^{2}+\mathrm{d} z^{2}\right) .
$$


The canonical variables that resemble the Ashtekar variables in full LQC are

$$
p=a^{2}, \quad c=\frac{\dot{a}}{2 a},
$$

which have

$$
\{c, p\}=1 \text {. }
$$

In the case of loop quantum cosmology, $p$ is allowed to actually take values in $\mathbb{R}$, so as to allow for a change in orientation. Note that this normalization differs from the literature by a factor of $8 \pi \gamma G_{N} / 3$ [2].

The constraint of the system is given by

$$
C=-24 \operatorname{sgn}(\mathrm{p}) \cdot \sqrt{|\mathrm{p}|} \mathrm{c}^{2}+3 \Lambda{\sqrt{|\mathrm{p}|^{\frac{3}{2}}}}
$$

The kinematical Hilbert space $\mathcal{H}_{\text {kin }}$ of LQC is the space of almost periodic functions in $c$, which has the functions

$$
|v\rangle:=c \longmapsto \mathrm{e}^{\mathrm{i} \frac{\nu}{2} c}, \quad \nu \in \mathbb{R}
$$

as a basis. So $\mathcal{H}_{\text {kin }}$ is not separable, since it has an uncountable basis, a feature that it shares with the Hilbert space of full loop quantum gravity. The quantization of $p$ acts on $\mathcal{H}_{\text {kin }}$ via differentiation by $c$ :

$$
\hat{p}|v\rangle=\frac{v}{2}|v\rangle,
$$

whereas $c$ is not definable as an operator. Rather, one has to employ $\exp (\mathrm{i} \lambda \hat{c})$ for all $\lambda \in \mathbb{R}$ as multiplication with the corresponding function of $c$, via:

$$
\mathrm{e}^{\mathrm{i} \lambda \hat{c}}|\nu\rangle=|\lambda+2 \nu\rangle,
$$

which shows that the failing weak continuity of $\lambda \mapsto \exp (i \lambda \hat{c})$ is the reason why one cannot define $\hat{c}$ without the exponential. This amounts to a problem when attempting to define the quantization of (3.4), since it explicitly contains $c$. This problem occurs in a similar fashion in the full theory, and it is resolved in LQC by introducing a parameter $\mu_{0}$ and approximating $c^{2}$ by $\mu_{0}^{-2} \sin ^{2}\left(\mu_{0} c\right) .^{3}$

If $c \ll 1 / \mu_{0}$, the two functions are quite similar, thus, if $\mu_{0}$ is sufficiently small, the function will be a good substitute for a large part of phase space. The latter function, however, can be quantized on $\mathcal{H}_{\text {kin }}$, which results in the following constraint operator:

$$
\hat{C}=-24\left(\frac{\sin \mu_{0} \hat{c}}{\mu_{0}}\right)^{2} \operatorname{sgn} \hat{\mathrm{p}} \widehat{\sqrt{|\mathrm{p}|}}+3 \Lambda \widehat{\sqrt{|\mathrm{p}|}}^{\frac{3}{2}},
$$

where

$$
\operatorname{sgn} \hat{\mathrm{p}} \widehat{\sqrt{|\mathrm{p}|}}|v\rangle=\frac{\left|v+\mu_{0}\right|^{\frac{3}{2}}-\left|v-\mu_{0}\right|^{\frac{3}{2}}}{3 \mu_{0}}|v\rangle
$$

is the quantization of $\operatorname{sgn} \mathrm{p} \sqrt{|\mathrm{p}|}$, that results from the attempt to keep the quantization scheme as close as possible to the ones employed in loop quantum gravity.

The introduction of the scale $\mu_{0}$ induces a splitting:

$$
\mathcal{H}_{\text {kin }}=\bigoplus_{\delta \in\left[0,4 \mu_{0}\right)} \mathcal{H}_{\delta}
$$

where each $\mathcal{H}_{\delta}=\operatorname{span}\left\{|v\rangle, v \in \delta+4 \mu_{0} \mathbb{Z}\right\}$ is separable and left invariant by the constraint. Thus, one can calculate the rigging map for each sector $\delta \in\left[0,4 \mu_{0}\right)$ separately.

3 In full LQG the parameter $\mu_{0}$ is not necessary, because spatial diffeomorphism invariance 'swallows it up' there. In LQC the spatial diffeomorphism group has been gauge-fixed so that one has to deal with $\mu_{0}$ as an artefact of the model. Note that recently [5] a method has been proposed of how to overcome this by replacing $\mu_{0}$ by an operator depending on $\hat{p}$, which cures this artefact and is more in the spirit of full LQG. 


\subsection{Solutions to the constraint}

We attempt to calculate the physical inner product for the above cosmological model with $\Lambda=0$. For this, we first use a trick that has been employed in [14] to compute the physical inner product for the case with $\Lambda \neq 0$ of Riemannian cosmology. For this case the 0 is in the pure point spectrum of the constraint, and hence the eigenvalue problem could be solved explicitly. It was reasoned that this stays true for the case of Lorentzian cosmology with nonzero cosmological constant. If $\Lambda$ is set to zero, then Riemannian and Lorentzian cosmology become indistinguishable, and then 0 is no longer in the pure point spectrum of $\hat{C}$. Still, the trick used in [14] will make it possible to compute the physical Hilbert space analytically.

Since the trick is in principle not applicable in full loop quantum gravity, we will also try to calculate the physical inner product without using it. Furthermore, since without the trick the constraint operator is no longer symmetric, we work with the master constraint operator $\hat{M}=\hat{C}^{\dagger} \hat{C}$ instead. In this setting, the master constraint operator will be too complicated to derive the physical inner product analytically, but the coherent states will enable us to approximate it well enough. This is precisely what one expects also in full LQG. In particular, we will find the approximate solutions are close to the solutions previously obtained analytically, which will show that the coherent states are in principle useful to approximate physical inner products. It will also demonstrate that, although the original constraint operator $\hat{C}$ has been replaced by $\hat{M}$, which has potentially many more solutions, the physical Hilbert space computed from $\hat{M}$ consists of solutions of $\hat{C}$ only.

\subsection{Riemannian cosmology with and without cosmological constant}

We briefly repeat the results of [14]: there, the Hilbert space was the same as the one used in the Lorentzian case we are looking at, but the constraint looked different. In particular, it was given classically by

$$
C_{\text {Riemann }}=24 \operatorname{sgn} \mathrm{p} \cdot \sqrt{|\mathrm{p}|} \mathrm{c}^{2}+3 \Lambda \sqrt{|\mathrm{p}|^{\frac{3}{2}}} .
$$

The trick is to modify this constraint by multiplication with $|p|^{-\frac{1}{2}}$, to arrive at the (up to the points $p=0$ ) classically equivalent function

$$
C_{\text {Riemann }}=24 c^{2}+3 \Lambda p \text {. }
$$

By introducing a length parameter $\mu_{0}$, as shown in section (3), this function can be quantized on $\mathcal{H}_{\text {kin }}$ as

$$
\hat{C}_{\text {Riemann }}=24\left(\frac{\sin \mu_{0} \hat{c}}{\mu_{0}}\right)^{2}+3 \Lambda \hat{p},
$$

from which the physical inner product was computed in [14]. The only solution to (3.12), which has nonzero physical norm, was calculated to be

$$
l=\sum_{\lambda \in-\frac{6}{\Lambda \mu_{0}^{2}}+4 \mu_{0} \mathbb{Z}} J_{\frac{\lambda}{4 \mu_{0}}-\frac{3}{2 \Lambda \mu_{0}^{3}}}\left(\frac{3}{2 \Lambda \mu_{0}^{3}}\right)\langle\lambda|,
$$

where $J_{n}(x)$ is the $n$th Bessel function of first kind. In particular, although there exist solutions to the constraint in every sector $\delta \in\left[0,4 \mu_{0}\right)$, only the solution in the sector $\delta=4 \mu_{0}\left[1-\bmod \left(\frac{3}{2 \Lambda \mu_{0}^{3}}, 1\right)\right]$ has nonzero physical norm. Here, $\bmod (x, y)$ denotes the remainder of $x / y$. So by the constraint, one sector is allowed, and the resulting physical Hilbert space is one-dimensional, as assumed for a theory with zero degrees of freedom. 
If one lets $\Lambda \rightarrow 0$, neither does the solution (3.14) converge, nor does the sector $\delta$ in which it is defined. So, in order to investigate the case $\Lambda=0$, we have to calculate the physical inner product explicitly, instead of taking the limit $\Lambda \rightarrow 0$ of the already known solutions. Classically, this amounts to

$$
C=24 c^{2}
$$

Since the quantized constraint

$$
\hat{C}=24 \frac{\sin ^{2} \mu_{0} \hat{c}}{\mu_{0}^{2}}
$$

is self-adjoint, we do not employ the master constraint, but calculate the rigging map (2.19) directly with $\hat{C}$. We have to compute (2.19)

$$
\eta[|v\rangle]|\mu\rangle=\lim _{t \rightarrow 0} \frac{\left\langle\mu\left|\mathrm{e}^{-\frac{\hat{C}^{2}}{t}}\right| v\right\rangle}{\left\langle v_{0}\left|\mathrm{e}^{-\frac{\hat{C}^{2}}{t}}\right| \nu_{0}\right\rangle},
$$

where $\left|v_{0}\right\rangle$ is a reference vector for correct normalization.

Since $\hat{C}$ is bounded, we have

$$
\begin{aligned}
\left\langle\nu\left|\mathrm{e}^{-\frac{\hat{c}^{2}}{t}}\right| \mu\right\rangle & =\sum_{n=0}^{\infty} \frac{(-1)^{n}}{n !} \frac{1}{t^{n}}\left\langle\mu\left|\hat{C}^{2 n}\right| \nu\right\rangle \\
& =\sum_{n=0}^{\infty} \frac{(-1)^{n}}{n !}\left(\frac{24}{\mu_{0}^{2} \sqrt{t}}\right)^{2 n}\left\langle\mu\left|\sin ^{4 n}\left(\mu_{0} \hat{c}\right)\right| v\right\rangle .
\end{aligned}
$$

With definition of $\mathrm{e}^{\mathrm{i} \mu_{0} \hat{c}}$ one can see that

$\left\langle\mu\left|\sin ^{4 n}\left(\mu_{0} \hat{c}\right)\right| \nu\right\rangle= \begin{cases}\frac{1}{16^{n}}(-1)^{\frac{\nu-\mu}{4 \mu_{0}}}\left(\begin{array}{c}4 n \\ 2 n+\frac{\nu-\mu}{4 \mu_{0}}\end{array}\right) & \text { for } \frac{\nu-\mu}{4 \mu_{0}} \in \mathbb{Z} \\ 0 & \text { else. }\end{cases}$

So in what follows, assume $\frac{\nu-\mu}{4 \mu_{0}}$ to be integer. Then we have

$$
\begin{aligned}
\left\langle v\left|\mathrm{e}^{-\frac{\hat{c}^{2}}{t}}\right| \mu\right\rangle & =(-1)^{\frac{v-\mu}{4 \mu_{0}}} \sum_{n=0}^{\infty} \frac{(-1)^{n}}{n !}\left(\frac{6}{\mu_{0}^{2} \sqrt{t}}\right)^{2 n}\left(\begin{array}{c}
4 n \\
2 n+\frac{\nu-\mu}{4 \mu_{0}}
\end{array}\right) \\
& =(-1)^{\frac{v-\mu}{4 \mu_{0}}} \sum_{n=0}^{\infty} \frac{(-1)^{n}}{n !}\left(\frac{6}{\mu_{0}^{2} \sqrt{t}}\right)^{2 n} \frac{\Gamma(4 n+1)}{\Gamma\left(2 n+1+\frac{v-\mu}{4 \mu_{0}}\right) \Gamma\left(2 n+1-\frac{v-\mu}{4 \mu_{0}}\right)} \\
& =(-1)^{N} \sum_{n=0}^{\infty} \frac{(-1)^{n}}{n !} a^{2 n} \frac{\Gamma(4 n+1)}{\Gamma(2 n+1+N) \Gamma(2 n+1-N)}
\end{aligned}
$$

with the abbreviations

$$
N=\frac{\nu-\mu}{4 \mu_{0}}, \quad a=\frac{6}{\mu_{0}^{2} \sqrt{t}} .
$$

Now we use the identity

$$
\sum_{n=0}^{\infty} \frac{(-1)^{n}}{n !} f(n)=\frac{1}{2 \pi \mathrm{i}} \int_{-\mathrm{i} \infty}^{\mathrm{i} \infty} \mathrm{d} s \Gamma(-s) f(s),
$$


(see, e.g., [1], p 559), for $f$ being as in (3.18). A coordinate transformation $s=-\frac{r+1}{4}$ yields

$$
\begin{aligned}
\sum_{n=0}^{\infty} \frac{(-1)^{n}}{n !} a^{2 n} & \frac{\Gamma(4 n+1)}{\Gamma(2 n+1+N) \Gamma(2 n+1-N)} \\
& =\frac{1}{2 \pi \mathrm{i}} \int_{-\mathrm{i} \infty}^{\mathrm{i} \infty} \mathrm{d} s \Gamma(-s) a^{2 s} \frac{\Gamma(4 s+1)}{\Gamma(2 s+1+N) \Gamma(2 s+1-N)} \\
& =-\frac{1}{8 \pi \mathrm{i}} \int_{\mathrm{i} \infty+1}^{-\mathrm{i} \infty+1} \mathrm{~d} r \Gamma(-r) a^{-\frac{r+1}{2}} \frac{\Gamma\left(\frac{r+1}{4}\right)}{\Gamma\left(\frac{1}{2}-\frac{r}{4}+N\right) \Gamma\left(\frac{1}{2}-\frac{r}{4}-N\right)}
\end{aligned}
$$

Using again (3.19), we get

$$
\begin{aligned}
\sum_{n=0}^{\infty} \frac{(-1)^{n}}{n !} a^{2 n} & \frac{\Gamma(4 n+1)}{\Gamma(2 n+1+N) \Gamma(2 n+1-N)} \\
& =\frac{1}{4} a^{-\frac{1}{2}} \sum_{n=0}^{\infty} \frac{(-1)^{n}}{n !} a^{-\frac{n}{2}} \frac{\Gamma\left(\frac{n+1}{4}\right)}{\Gamma\left(\frac{1}{2}-\frac{n}{4}+N\right) \Gamma\left(\frac{1}{2}-\frac{n}{4}-N\right)} .
\end{aligned}
$$

So, finally

$\left\langle v\left|\mathrm{e}^{-\frac{\hat{c}^{2}}{t}}\right| \mu\right\rangle=\frac{(-1)^{\frac{v-\mu}{4 \mu_{0}}}}{4}\left(\frac{\mu_{0}^{2} \sqrt{t}}{6}\right)^{\frac{1}{2}} \sum_{n=0}^{\infty} \frac{(-1)^{n}}{n !}\left(\frac{\mu_{0}^{2} \sqrt{t}}{6}\right)^{\frac{n}{2}} \frac{\Gamma\left(\frac{n+1}{4}\right)}{\Gamma\left(\frac{1}{2}-\frac{n}{4}+\frac{v-\mu}{4 \mu_{0}}\right) \Gamma\left(\frac{1}{2}-\frac{n}{4}-\frac{v-\mu}{4 \mu_{0}}\right)}$.

With this, (2.19) and

$$
\frac{1}{\Gamma\left(\frac{1}{2}+N\right) \Gamma\left(\frac{1}{2}-N\right)}=\frac{1}{\pi}(-1)^{N}
$$

we get

$$
\eta[|v\rangle]|\mu\rangle=\lim _{t \rightarrow 0} \frac{\left\langle\nu\left|\mathrm{e}^{-\frac{\hat{C}^{2}}{t}}\right| \mu\right\rangle}{\left\langle v_{0}\left|\mathrm{e}^{-\frac{\hat{c}^{2}}{t}}\right| \nu_{0}\right\rangle}=1
$$

if $\frac{\nu-\mu}{4 \mu_{0}}$ is an integer, 0 else for any $\left|\nu_{0}\right\rangle \in \mathcal{H}_{\text {kin }}$. Since the result does not depend on the choice of $\left|v_{0}\right\rangle$, we might take any one.

From this we see the following: the physical Hilbert space is not separable, it consists of the linear forms

$$
l_{\delta}:=\sum_{\lambda \in \delta+4 \mu_{0} \mathbb{Z}}\langle\lambda|, \quad \delta \in\left[0,4 \mu_{0}\right)
$$

with the physical inner product

$$
\left\langle l_{\delta} \mid l_{\varepsilon}\right\rangle_{\text {phys }}=\delta_{\delta \varepsilon} .
$$

So it decomposes into uncountably many one-dimensional, orthogonal subspaces:

$$
\mathcal{H}_{\text {phys }}=\bigoplus_{\delta \in\left[0,4 \mu_{0}\right)} \mathbb{C}
$$

This shows an important fact: although the classical system under consideration has zero degrees of freedom, the corresponding physical Hilbert space $\mathcal{H}_{\text {phys }}$ of the quantum theory has uncountably many dimensions. Also, the theory is not superselected, since the operators

$$
\hat{O}_{\lambda}:=\sin (\lambda \hat{c}), \quad \lambda>0
$$

are self-adjoint (with respect to the physical inner product) and commute with the constraint, hence turn into Dirac observables on the physical Hilbert space. These observables act irreducibly in $\mathcal{H}_{\text {phys }}$, hence the physical Hilbert space is not superselected. 
The fact that quantum theory behaves so significantly different from the classical one can be traced back to the quantization procedure. Since the constraint, the phase-space function $c^{2}$, has no quantization on $\mathcal{H}_{\text {kin }}$, one has to come up with a different operator and chooses $\mu_{0}^{-2} \sin ^{2} \mu_{0} \hat{c}$ for some fixed $\mu_{0}$. This $\mu_{0}$ introduces a scale into the theory that has not been there before. Had one chosen the uncountable set of constraints $\lambda^{-2} \sin ^{2} \lambda \hat{c}$ for $\lambda>0$, which is classically equivalent to $c=0$, then the only solutions for this system would have been the linear forms

$$
l_{0}=\sum_{\nu \in \mathbb{R}}\langle\nu|, \quad l_{1}=\sum_{n \in \mathbb{R}} v\langle v| .
$$

In this case the space of solutions to the constraints had been two-dimensional right from the beginning. To conclude which of these two solutions is the physically correct one, one would, of course, need to define a physical inner product.

Note that the unphysically large size of $\mathcal{H}_{\text {phys }}$ should be considered as an artefact of the model, resulting from the introduction of the scale $\mu_{0}$ to a formerly scale-invariant theory, i.e. going over from (3.15) to (3.16). This feature does not occur if the model already contains scales, i.e. determined by massive matter fields or the cosmological constant $\Lambda$ (as, for instance in [14], where the physical Hilbert space is one-dimensional, as expected). Still, the results, of course, depend on the relation of the scales from the theory and the scale from $\mu_{0}$, as one can see i.e. in (3.14).

\subsection{The master constraint}

The computation of the physical inner product in the case of vanishing cosmological constant has been possible explicitly, because the constraint (3.11) had been changed into the classically equivalent (3.12). Unfortunately, this trick is not available for the full theory, for an important reason: to become an honest operator in full loop quantum gravity, the density weight of a phase-space function has to be +1 ! But multiplying with a nonzero power of the triads $E_{i}^{a}$ (which correspond to $p$ in the cosmological case) changes the density weight, hence destroys the quantization scheme. The honest constraint is therefore

$$
\hat{C}=\left(\frac{\sin \mu_{0} \hat{c}}{\mu_{0}}\right)^{2} \operatorname{sgn} \hat{\mathrm{p}} \widehat{\sqrt{|\mathrm{p}|}} .
$$

We would like to consider this constraint instead of (3.16). In this form, the physical inner product can no longer be calculated explicitly, but we will show how the complexifier coherent states will be able to provide an approximation scheme for it.

We start by giving all solutions of the constraint equation

$$
l \circ \hat{C}^{\dagger}=0 .
$$

In particular, the solutions are, for $\delta \in\left(0,4 \mu_{0}\right)$ :

$$
l_{(a, b)}=\sum_{\lambda \in \delta+4 \mu_{0} \mathbb{Z}} \frac{a+b \lambda}{\left|\lambda+\mu_{0}\right|^{\frac{3}{2}}-\left|\lambda-\mu_{0}\right|^{\frac{3}{2}}}\langle\lambda|, \quad a, b \in \mathbb{C},
$$

whereas the solutions in the sector $\delta=0$ are the following:

$$
l_{(a, b)}=\sum_{\lambda \in \delta+4 \mu_{0} \mathbb{Z}} \frac{a \lambda}{\left|\lambda+\mu_{0}\right|^{\frac{3}{2}}-\left|\lambda-\mu_{0}\right|^{\frac{3}{2}}}\langle\lambda|+b\langle 0|, \quad a, b \in \mathbb{C} .
$$

Note that the space of solutions is two-dimensional in each case, consisting of one bounded and one unbounded solution. This means that the sets of coefficients $l_{(a, b)}|\lambda\rangle$ in $(3.25)$ 
and (3.26) are either bounded or unbounded. The bounded solutions are normalizable in the kinematical inner product for the case $\delta=0$ only!

The master constraint is quite simple, since there is only one constraint:

$$
\hat{M}=\hat{C}^{\dagger} \hat{C}
$$

i.e.

$$
\hat{M}=\widehat{\sqrt{|p|}} \operatorname{sgn} \hat{\mathrm{p}}\left(\frac{\sin \mu_{0} \hat{\mathrm{c}}}{\mu_{0}}\right)^{4} \operatorname{sgn} \hat{\mathrm{p}} \widehat{\sqrt{|\mathrm{p}|}} .
$$

From this form it is immediately clear that the solutions (3.25) and (3.26) satisfy

$$
l_{(a, b)} \circ \hat{M}=0 .
$$

Still, there are more solutions to (3.29). These are, in particular, for $\delta \in\left(0,4 \mu_{0}\right)$ :

$$
\tilde{l}_{(c, d)}=\sum_{\lambda \in \delta+4 \mu_{0} \mathbb{Z}} \frac{c \lambda^{2}+d \lambda^{3}}{\left|\lambda+\mu_{0}\right|^{\frac{3}{2}}-\left|\lambda-\mu_{0}\right|^{\frac{3}{2}}}\langle\lambda|, \quad c, d \in \mathbb{C} .
$$

The linear forms (3.30) solve (3.29), but not (3.24). So, as one would have guessed from the outset, there are more solutions to the master constraint than to the original constraint, and it is common belief that the additional solutions will turn out to be spurious, that is, have zero norm in the physical inner product yet to be computed.

The form of the master constraint (3.28) is too complicated to give a closed expression for $\left\langle\nu\left|\hat{M}^{2 n}\right| \mu\right\rangle$, which is needed in order to compute the rigging map (2.19). It is at this point, where the coherent states come into play, since they allow for an approximation of (3.28), as will be shown in the following.

\subsection{Coherent states}

The complexifier coherent states were defined in [10-12] and their properties were investigated in $[21,23-25]$, where their particular use for approximations was pointed out. We will use the simplest of these states, in particular the ones for the gauge group $U(1)$. They are defined on each $\mathcal{H}_{\delta}=\operatorname{span}\left\{|\lambda\rangle, \lambda \in \delta+4 \mu_{0} \mathbb{Z}\right\}$, with $\delta \in\left[0,4 \mu_{0}\right)$, by

$$
|u\rangle=\sum_{\lambda \in \delta+4 \mu_{0} \mathbb{Z}} \mathrm{e}^{-\lambda^{2} \frac{s^{2}}{2}} \mathrm{e}^{\mathrm{i} \lambda u}|\lambda\rangle .
$$

The complex number $u=c-\mathrm{i} p$ that labels the coherent states resembles the phase-space coordinates. With our conventions here, the states are peaked in phase space around the points $\left(-2 c, p / s^{2}\right)$ with width $s$. Hence, $s$ plays the role of a 'classicality parameter'.

The coherent states have a number of properties that make them useful for approximations. In particular, they provide a resolution of unity via

$$
\frac{\mu_{0}}{\sqrt{\pi t}} \int_{-\frac{\pi}{\mu_{0}}}^{\frac{\pi}{\mu_{0}}} \frac{\mathrm{d} c}{2 \pi} \int_{\mathbb{R}} \mathrm{d} p \mathrm{e}^{-\frac{p^{2}}{s^{2}}}|c-\mathrm{i} p\rangle\langle c-\mathrm{i} p|=\mathrm{id}_{\mathcal{H}_{\delta}} .
$$

Furthermore, the coherent states approximate classical states in the following sense: given a phase-space function $f(c, p)$, the expectation value of the operator $\hat{f}:=f(\hat{c}, \hat{p})$ in a coherent state $|c-\mathrm{i} p\rangle$ is, up to corrections in $t$, given by

$$
\frac{\langle c-\mathrm{i} p|\hat{f}| c-\mathrm{i} p\rangle}{\langle c-\mathrm{i} p \mid c-\mathrm{i} p\rangle} \approx f\left(-2 c, p / s^{2}\right),
$$

where the quality of the approximation depends on $f$, and will in general not be equally good for all phase-space functions [25]. 
To calculate the rigging map corresponding to the master constraint $M$ according to (2.19), we need to calculate

$$
\left\langle\nu\left|\mathrm{e}^{-\frac{\hat{M}^{2}}{t}}\right| \mu\right\rangle:=\sum_{n=0}^{\infty} \frac{1}{n !}\left(\frac{-1}{t}\right)^{n}\left\langle, \nu\left|\hat{M}^{2 n}\right| \mu\right\rangle,
$$

which exists, since the basis vectors can be shown to be analytic vectors for $\hat{M}$. Because of (3.34) we need to know matrix elements of even powers of $\hat{M}$ in arbitrary basis states $|v\rangle,|\mu\rangle$. This cannot be done analytically, due to the inconvenient structure of (3.9) in the operator $\hat{M}$, but the coherent states will provide a way of approximating $\left\langle\nu\left|\hat{M}^{2 n}\right| \mu\right\rangle$, in the following way.

With $f=M^{2}, M$ being the master constraint of our model, we will find a generalization of (3.33), in particular

$\frac{\left\langle c^{\prime}-\mathrm{i} p^{\prime}|\hat{f}| c-\mathrm{i} p\right\rangle}{\left\langle c^{\prime}-\mathrm{i} p^{\prime} \mid c-\mathrm{i} p\right\rangle} \approx \tilde{f}\left(-\left(c+c^{\prime}\right)+\mathrm{i}\left(p-p^{\prime}\right), \frac{\left(p+p^{\prime}\right)+\mathrm{i}\left(c-c^{\prime}\right)}{2 s^{2}}\right)$,

where $\tilde{f}$ is the analytical continuation of $f$ in both variables. This continuation is possible for $f=M^{2}$, since $M^{2}$ is in fact real analytic in both variables. Hence, to approximate $\left\langle\nu\left|\hat{M}^{2}\right| \mu\right\rangle$, we first compute the matrix elements of $\hat{M}^{2}$ in coherent states $\left\langle u\left|\hat{M}^{2}\right| v\right\rangle$, with $u=c_{1}-\mathrm{i} p_{1}, v=c_{2}-\mathrm{i} p_{2}$, and then use (3.32) to write

$$
\left\langle\nu\left|\hat{M}^{2}\right| \mu\right\rangle=\frac{\mu_{0}^{2}}{\pi t} \int_{\left[-\frac{\pi}{\mu_{0}}, \frac{\pi}{\mu_{0}}\right]^{2}} \frac{\mathrm{d} c_{1}}{2 \pi} \frac{\mathrm{d} c_{2}}{2 \pi} \int_{\mathbb{R}^{2}} \mathrm{~d} p_{1} \mathrm{~d} p_{2} \mathrm{e}^{-\frac{p_{1}^{2}+p_{2}^{2}}{s^{2}}}\langle\nu \mid v\rangle\left\langle v\left|\hat{M}^{2}\right| u\right\rangle\langle u \mid \mu\rangle .
$$

This will enable us to develop an approximation of $\left\langle\nu\left|\hat{M}^{2}\right| \mu\right\rangle$. After that, we will employ the completeness of the $|\nu\rangle$-basis on $\mathcal{H}_{\delta}$ to compute

$$
\left\langle\nu\left|\hat{M}^{2 n}\right| \mu\right\rangle=\sum_{\lambda_{1}, \lambda_{2}, \cdots, \lambda_{n-1} \in \delta+4 \mu_{0} \mathbb{Z}}\left\langle\nu\left|\hat{M}^{2}\right| \lambda_{1}\right\rangle\left\langle\lambda_{1}\left|\hat{M}^{2}\right| \lambda_{2}\right\rangle \cdots\left\langle\lambda_{n-1}\left|\hat{M}^{2}\right| \mu\right\rangle .
$$

Since there is no closed expression for the result available, we will use another approximation in order to arrive at a tangible expression for $\left\langle\nu\left|\hat{M}^{2 n}\right| \mu\right\rangle$, the quality of which will depend on $v, \mu$, making the approximation phase space dependent. Afterwards, we will plug these approximations for $\left\langle\nu\left|\hat{M}^{2 n}\right| \mu\right\rangle$ into (3.34) and investigate the limit $t \rightarrow 0$, in order to arrive at a final expression for the rigging map (2.19).

\section{Computation of $\left\langle\nu\left|\hat{M}^{2}\right| \mu\right\rangle$}

\subsection{Expectation value of the master constraint in coherent states}

With

$$
\operatorname{sgn} \hat{\mathrm{p}} \widehat{\sqrt{|\mathrm{p}|}}|v\rangle=\frac{\left|v+\mu_{0}\right|^{\frac{3}{2}}-\left|v-\mu_{0}\right|^{\frac{3}{2}}}{3 \mu_{0}}|v\rangle=: \rho_{\nu}|v\rangle
$$

the action of the squared master constraint on a vector $|\nu\rangle$ is, via (3.28), given by

$$
\begin{aligned}
\hat{M}^{2}|v\rangle=\frac{1}{256} & {\left[\rho_{v} \rho_{v+8 \mu_{0}}^{2} \rho_{v+16 \mu_{0}}\left|v+16 \mu_{0}\right\rangle-4 \rho_{\nu} \rho_{v+12 \mu_{0}}\left(\rho_{v+8 \mu_{0}}^{2}+\rho_{v+4 \mu_{0}}^{2}\right)\left|v+12 \mu_{0}\right\rangle\right.} \\
& +\rho_{\nu} \rho_{v+8 \mu_{0}}\left(6 \rho_{v+8 \mu_{0}}^{2}+16 \rho_{v+4 \mu_{0}}^{2}+6 \rho_{v}^{2}\right)\left|v+8 \mu_{0}\right\rangle \\
& -\rho_{v} \rho_{v+4 \mu_{0}}\left(4 \rho_{v+8 \mu_{0}}^{2}+24 \rho_{v+4 \mu_{0}}^{2}+24 \rho_{v}^{2}+4 \rho_{v-4 \mu_{0}}^{2}\right)\left|v+4 \mu_{0}\right\rangle \\
& +\rho_{v}^{2}\left(\rho_{v+8 \mu_{0}}^{2}+16 \rho_{v+4 \mu_{0}}^{2}+36 \rho_{v}^{2}+16 \rho_{v-4 \mu_{0}}^{2}+\rho_{v-8 \mu_{0}}^{2}\right)|v\rangle \\
& -\rho_{v} \rho_{v-4 \mu_{0}}\left(4 \rho_{v-8 \mu_{0}}^{2}+24 \rho_{v-4 \mu_{0}}^{2}+24 \rho_{v}^{2}+4 \rho_{v+4 \mu_{0}}^{2}\right)\left|v-4 \mu_{0}\right\rangle
\end{aligned}
$$




$$
\begin{aligned}
& +\rho_{\nu} \rho_{\nu-8 \mu_{0}}\left(6 \rho_{\nu-8 \mu_{0}}^{2}+16 \rho_{\nu-4 \mu_{0}}^{2}+6 \rho_{\nu}^{2}\right)\left|v-8 \mu_{0}\right\rangle \\
& \left.-4 \rho_{\nu} \rho_{\nu-12 \mu_{0}}\left(\rho_{\nu-8 \mu_{0}}^{2}+\rho_{\nu-4 \mu_{0}}^{2}\right)\left|v-12 \mu_{0}\right\rangle+\rho_{\nu} \rho_{\nu-8 \mu_{0}}^{2} \rho_{\nu-16 \mu_{0}}\left|v-16 \mu_{0}\right\rangle\right] .
\end{aligned}
$$

The coherent states in the sector $\delta \in\left[0,4 \mu_{0}\right)$ are given by

$$
|u\rangle=\sum_{\lambda \in \delta+4 \mu_{0} \mathbb{Z}} \mathrm{e}^{-\lambda^{2} \frac{s^{2}}{2}} \mathrm{e}^{\mathrm{i} \lambda u}|\lambda\rangle .
$$

As shown in [14], this state, labelled by the complex number $u=c-\mathrm{i} p$, is (in our notation) sharply peaked around the point $\left(-2 c, p / s^{2}\right)$ in phase space with width $s$. One gets, for two coherent states $|u\rangle,|v\rangle$ :

$$
\left\langle v\left|\hat{M}^{2}\right| u\right\rangle=\sum_{\text {terms }} \sum_{v, \mu \in \delta+4 \mu_{0} \mathbb{Z}} \mathrm{e}^{-\left(v^{2}+\mu^{2}\right) \frac{s^{2}}{2}} \mathrm{e}^{\mathrm{i}(v u-\mu \bar{v})} \rho_{\mu+a \mu_{0}} \rho_{\mu+b \mu_{0}} \rho_{\mu+c \mu_{0}} \rho_{\mu+\mathrm{d} \mu_{0}} \delta_{v, \mu+2 e \mu_{0}}
$$

with integer numbers $a, b, \ldots, e$. In order to evaluate this, we write

$$
\rho_{x+a \mu_{0}} \rho_{x+b \mu_{0}} \rho_{x+c \mu_{0}} \rho_{x+\mathrm{d} \mu_{0}}=\left(x+m^{\prime} \mu_{0}\right)^{2}+\mu_{0}^{2} \Delta+R(x)
$$

where

$$
m^{\prime}=\frac{a+b+c+d}{4}, \quad \Delta=-\frac{1}{6}-\frac{1}{16}(a+b+c+d)^{2}-\frac{a^{2}+b^{2}+c^{2}+d^{2}}{4}
$$

and $\lim _{|x| \rightarrow \infty} x R(x)<\infty$. If we neglect $R(x)$, we get

$$
\begin{aligned}
\left\langle v\left|\hat{M}^{2}\right| u\right\rangle \approx & \sum_{\text {terms }} \sum_{\lambda} \mathrm{e}^{-\lambda^{2} s^{2}} \mathrm{e}^{-\left(e m_{0}\right)^{2} s^{2}} \mathrm{e}^{\mathrm{i} \lambda(u-\bar{v})} \mathrm{e}^{\mathrm{i}\left(e \mu_{0}\right)(u+\bar{v})}\left[\left(\lambda+m \mu_{0}\right)^{2}+\mu_{0}^{2} \Delta\right] \\
= & \sum_{\text {terms }} \exp \left(-\left(\frac{u+\bar{v}}{2 s^{2}}\right)^{2} s^{2}\right) \exp \left(-\left(\frac{u-\bar{v}}{2 s^{2}}\right)^{2} s^{2}\right) \exp \left(-\left(\mathrm{i} \frac{u+\bar{v}}{2 s^{2}}-e m_{0}\right)^{2} s^{2}\right) \\
& \times \sum_{\lambda \in \delta+4 \mu_{0} \mathbb{Z}} \exp \left(-\left(\lambda-\mathrm{i} \frac{u-\bar{v}}{2 s^{2}}\right)^{2} s^{2}\right)\left[\left(\lambda+m \mu_{0}\right)^{2}+\mu_{0}^{2} \Delta\right],
\end{aligned}
$$

with $m=m^{\prime}-e$. Applying the Poisson summation formula we get

$$
\begin{aligned}
\sum_{\lambda \in \delta+\mu_{0} \mathbb{Z}} \mathrm{e}^{-\left(\lambda-\mathrm{i} \frac{u-\bar{v}}{2 s^{2}}\right)^{2} s^{2}}\left(\lambda+m \mu_{0}\right)^{2}= & \frac{1}{\mu_{0}} \sum_{n \in \mathbb{Z}} \exp \left(\frac{2 \pi n \delta i}{\mu_{0}}\right) \int \mathrm{d} x \exp \left(-\mathrm{i} \frac{2 \pi n}{\mu_{0}} x\right) \\
& \times \exp \left(-\left(x-\mathrm{i} \frac{u-\bar{v}}{2 s^{2}}\right)^{2} s^{2}\right)\left[\left(x+m \mu_{0}\right)^{2}+\mu_{0}^{2} \Delta\right] \\
= & \frac{1}{\mu_{0}} \sqrt{\frac{\pi}{s^{2}}} \exp \left(\left(\frac{u-\bar{v}}{2 s^{2}}\right)^{2} s^{2}\right) \exp \left(-\left(\frac{u-\bar{v}}{2 s^{2}}-\frac{\pi n}{\mu_{0} s^{2}}\right)^{2} s^{2}\right) \\
& \times\left[\left(\mathrm{i} \frac{u-\bar{v}}{2 s^{2}}-\mathrm{i} \frac{\pi n}{\mu_{0} s^{2}}+m \mu_{0}\right)^{2}+\frac{1}{2 s^{2}}+\mu_{0}^{2} \Delta\right] .
\end{aligned}
$$

Reinserting this into (4.5) we thus obtain

$$
\begin{aligned}
\left\langle v\left|\hat{M}^{2}\right| u\right\rangle \approx \frac{1}{\mu_{0}} & \sqrt{\frac{\pi}{s^{2}}} \sum_{n \in \mathbb{Z}} \exp \left(\frac{2 \pi n \delta \mathrm{i}}{\mu_{0}}\right) \exp \left(-\left(\frac{u-\bar{v}}{2 s^{2}}-\frac{\pi n}{\mu_{0} s^{2}}\right)^{2} s^{2}\right) \\
& \times \sum_{\text {terms }} \mathrm{e}^{\mathrm{i} e \mu_{0}(u+\bar{v})} \mathrm{e}^{-\left(e \mu_{0}\right)^{2} s^{2}}\left[\left(\mathrm{i} \frac{u-\bar{v}}{2 s^{2}}-i \frac{\pi n}{\mu_{0} s^{2}}\right)^{2}\right. \\
& \left.+\frac{1}{s^{2}}\left(\frac{1}{2}+2 \mathrm{i}\left(\frac{u-\bar{v}}{2 s^{2}}-\frac{\pi n}{\mu_{0} s^{2}}\right) m \mu_{0}\right)+\mu_{0}^{2}\left(m^{2}+\Delta\right)\right] .
\end{aligned}
$$


Calculating the sum over the different terms explicitly, taking the prefactors in (4.1) into account, we obtain

$$
\begin{aligned}
& \sum_{\text {terms }} \mathrm{e}^{\mathrm{i} e \mu_{0}(u+\bar{v})} \mathrm{e}^{-\left(e \mu_{0}\right)^{2} s^{2}}=\mathrm{e}^{s^{2} \frac{\partial^{2}}{\partial z^{2}}} \mid z=\mathrm{i}(u+\bar{v}) \mu_{0} \sin ^{8} z \\
& \sum_{\text {terms }} \mathrm{e}^{\mathrm{i} e \mu_{0}(u+\bar{v})} \mathrm{e}^{-\left(e \mu_{0}\right)^{2} s^{2}} m=0 \\
& \sum_{\text {terms }} \mathrm{e}^{\mathrm{i} e \mu_{0}(u+\bar{v})} \mathrm{e}^{-\left(e \mu_{0}\right)^{2} s^{2}}\left(m^{2}+\Delta\right)=\frac{1}{16} \mathrm{e}^{s^{2} \frac{\partial^{2}}{\partial z^{2}}} \mid z=\mathrm{i}(u+\bar{v}) \mu_{0}\left(-\frac{193}{48} \cos 8 \mu_{0} z\right. \\
& \left.+\frac{109}{6} \cos 6 \mu_{0} z-\frac{487}{12} \cos 4 \mu_{0} z+\frac{55}{6} \cos 2 \mu_{0} z-\frac{35}{48}\right) \\
& =: \frac{1}{16} \mathrm{e}^{s^{2} \frac{\partial^{2}}{\partial z^{2}}} \mid z=\mathrm{i}(u+\bar{v}) \mu_{0} \zeta(z)
\end{aligned}
$$

So

$$
\begin{aligned}
\left\langle v\left|\hat{M}^{2}\right| u\right\rangle \approx \frac{1}{\mu_{0}} & \sqrt{\frac{\pi}{s^{2}}} \sum_{n \in \mathbb{Z}} \exp \left(\frac{2 \pi n \delta \mathrm{i}}{\mu_{0}}\right) \exp \left(-\left(\frac{u-\bar{v}}{2 s^{2}}-\frac{\pi n}{\mu_{0} s^{2}}\right)^{2} s^{2}\right) \\
& \times\left[\exp \left(s^{2} \frac{\partial^{2}}{\partial z^{2}}\right) \mid z=\mathrm{i}(u+\bar{v}) \mu_{0} \sin ^{8} z\left(\left(\mathrm{i} \frac{u-\bar{v}}{2 s^{2}}-\mathrm{i} \frac{\pi n}{\mu_{0} s^{2}}\right)^{2}+\frac{1}{2 s^{2}}\right)\right. \\
+ & \left.\frac{\mu_{0}^{2}}{16} \exp \left(s^{2} \frac{\partial^{2}}{\partial z^{2}}\right) \mid z=\mathrm{i}(u+\bar{v}) \mu_{0} \zeta(z)\right] .
\end{aligned}
$$

\subsection{Matrix elements of $\hat{M}^{2}$ with respect to the basis vectors}

We use the result from the last section to calculate now arbitrary matrix elements of $\hat{M}^{2}$. For this, we need the resolution of unity provided by the coherent states (3.32):

$$
\frac{\mu_{0}}{\sqrt{\pi s^{2}}} \int_{-\frac{\pi}{\mu_{0}}}^{\frac{\pi}{\mu_{0}}} \frac{\mathrm{d} c}{2 \pi} \int_{\mathbb{R}} \mathrm{d} p \mathrm{e}^{-\frac{p^{2}}{s^{2}}}|c-\mathrm{i} p\rangle\langle c-\mathrm{i} p|=\mathrm{id}_{\mathcal{H}_{\delta}} .
$$

so we have:

$\left\langle v\left|\hat{M}^{2}\right| \mu\right\rangle=\frac{\mu_{0}^{2}}{\pi s^{2}} \int_{\left[-\frac{\pi}{\mu_{0}}, \frac{\pi}{\mu_{0}}\right]^{2}} \frac{\mathrm{d} c_{1}}{2 \pi} \frac{\mathrm{d} c_{2}}{2 \pi} \int_{\mathbb{R}^{2}} \mathrm{~d} p_{1} \mathrm{~d} p_{2} \mathrm{e}^{-\frac{p_{1}^{2}+p_{2}^{2}}{s^{2}}}\langle v \mid v\rangle\left\langle v\left|\hat{M}^{2}\right| u\right\rangle\langle u \mid \mu\rangle$

with $u=c_{2}-\mathrm{i} p_{2}, v=c_{1}-\mathrm{i} p_{1}$. To make life simpler, we will split (4.8) up into several parts before inserting and perform the integrations separately. Expanding the first exponential derivative in (4.8) into a power series, we obtain

$$
\begin{aligned}
\left\langle v\left|\hat{M}^{2}\right| u\right\rangle=\frac{1}{\mu_{0}} & \sqrt{\frac{\pi}{s^{2}}} \sum_{n \in \mathbb{Z}} \exp \left(\frac{2 \pi n \delta \mathrm{i}}{\mu_{0}}\right) \exp \left(-\left(\frac{u-\bar{v}}{2 s^{2}}-\frac{\pi n}{\mu_{0} s^{2}}\right)^{2} s^{2}\right) \sin ^{8}\left(\mu_{0}(u+\bar{v})\right) \\
& \times\left(\left(\mathrm{i} \frac{u-\bar{v}}{2 s^{2}}-\mathrm{i} \frac{\pi n}{\mu_{0} s^{2}}\right)^{2}+\frac{1}{2 s^{2}}\right)+\frac{1}{\mu_{0}} \sqrt{\frac{\pi}{s^{2}}} \sum_{m=1}^{\infty} \frac{s^{2 m}}{m !} \frac{\partial^{2 m}}{\partial z^{2 m}}{ }_{\mid z=\left(\mu_{0}(u+\bar{v})\right)} \sin ^{8} z \\
& \times \sum_{n \in \mathbb{Z}} \exp \left(\frac{2 \pi n \delta \mathrm{i}}{\mu_{0}}\right) \exp \left(-\left(\frac{u-\bar{v}}{2 s^{2}}-\frac{\pi n}{\mu_{0} s^{2}}\right)^{2} s^{2}\right) \\
& \times\left(\left(\mathrm{i} \frac{u-\bar{v}}{2 s^{2}}-\mathrm{i} \frac{\pi n}{\mu_{0} s^{2}}\right)^{2}+\frac{1}{2 s^{2}}\right)+\frac{1}{\mu_{0}} \sqrt{\frac{\pi}{s^{2}}} \sum_{n \in \mathbb{Z}} \exp \left(\frac{2 \pi n \delta \mathrm{i}}{\mu_{0}}\right)
\end{aligned}
$$




$$
\times\left.\exp \left(-\left(\frac{u-\bar{v}}{2 s^{2}}-\frac{\pi n}{\mu_{0} s^{2}}\right)^{2} s^{2}\right) \frac{\mu_{0}^{2}}{16} \exp \left(s^{2} \frac{\partial^{2}}{\partial z^{2}}\right)\right|_{z=\mathrm{i}(u+\bar{v}) \mu_{0}} \zeta(z) .
$$

We insert now term by term of (4.11) into (4.10). We start with the zeroth order term of the derivative expansion:

$$
\begin{aligned}
\frac{\mu_{0}}{\sqrt{\pi s^{6}}} \int_{\left[-\frac{\pi}{\mu_{0}}, \frac{\pi}{\mu_{0}}\right]^{2}} & \frac{\mathrm{d} c_{1}}{2 \pi} \frac{\mathrm{d} c_{2}}{2 \pi} \int_{\mathbb{R}^{2}} \mathrm{~d} p_{1} \mathrm{~d} p_{2} \exp \left(-\left(v-\frac{p_{1}}{s^{2}}\right)^{2} \frac{s^{2}}{2}\right) \exp \left(-\left(\mu-\frac{p_{2}}{s^{2}}\right)^{2} \frac{s^{2}}{2}\right) \mathrm{e}^{\mathrm{i} v c_{1}} \mathrm{e}^{-\mathrm{i} \mu c_{2}} \\
& \times \exp \left(-\frac{p_{1}^{2}+p_{2}^{2}}{2 s^{2}}\right) \sin ^{8}\left[\mu_{0}\left(c_{1}+c_{2}-\mathrm{i}\left(p_{2}-p_{1}\right)\right)\right] \\
& \times \sum_{n \in \mathbb{Z}} \exp \left(\frac{2 \pi \mathrm{i} n \delta}{\mu_{0}}\right) \exp \left(\left(\frac{p_{2}+p_{1}-\mathrm{i}\left(c_{2}-c_{1}\right)}{2 s^{2}}+\mathrm{i} \frac{\pi n}{\mu_{0} s^{2}}\right)^{2} s^{2}\right) \\
& \times\left[\left(\frac{p_{2}+p_{1}-\mathrm{i}\left(c_{2}-c_{1}\right)}{2 s^{2}}+\mathrm{i} \frac{\pi n}{\mu_{0} s^{2}}\right)^{2}+\frac{1}{2 s^{2}}\right] .
\end{aligned}
$$

Since the exponentials and the sine function are all invariant under the substitution $c_{1} \rightarrow c_{1}+\frac{2 \pi}{\mu_{0}}$, this integral has the following form:

$$
\int_{-\frac{\pi}{\mu_{0}}}^{-\frac{\pi}{\mu_{0}}} \frac{\mathrm{d} c_{1}}{2 \pi} \sum_{n=0}^{\infty} f\left(c_{1}+\frac{2 \pi n}{\mu_{0}}\right) \mathrm{e}^{\frac{2 \pi \mathrm{i} n \delta}{\mu_{0}}}=\int_{\mathbb{R}} \frac{\mathrm{d} c_{1}}{2 \pi} f\left(c_{1}\right) \chi\left(c_{1}\right),
$$

where

$$
\chi\left(c_{1}\right):=\sum_{n=0}^{\infty} \chi_{\left[-\frac{\pi}{\mu_{0}}, \frac{\pi}{\mu_{0}}\right]}\left(c_{1}\right) \mathrm{e}^{\frac{2 \pi \mathrm{i} n \delta}{\mu_{0}}}
$$

is a sum over characteristic functions. This gives us

$$
\begin{aligned}
\frac{\mu_{0}}{\sqrt{\pi s^{6}}} \int_{\mathbb{R}} \frac{\mathrm{d} c_{1}}{2 \pi} \int_{-\frac{\pi}{\mu_{0}}}^{\frac{\pi}{\mu_{0}}} \frac{\mathrm{d} c_{2}}{2 \pi} \int_{\mathbb{R}^{2}} \mathrm{~d} p_{1} \mathrm{~d} p_{2} \exp \left(-\left(v-\frac{p_{1}}{s^{2}}\right)^{2} \frac{s^{2}}{2}\right) \exp \left(-\left(\mu-\frac{p_{2}}{s^{2}}\right)^{2} \frac{s^{2}}{2}\right) \mathrm{e}^{\mathrm{i} v c_{1}} \mathrm{e}^{-\mathrm{i} \mu c_{2}} \\
\quad \times \exp \left(-\frac{p_{1}^{2}+p_{2}^{2}}{2 s^{2}}\right) \sin ^{8}\left[\mu_{0}\left(c_{1}+c_{2}-\mathrm{i}\left(p_{2}-p_{1}\right)\right)\right] \chi\left(c_{1}\right) \\
\quad \times \exp \left(\left(\frac{p_{2}+p_{1}-\mathrm{i}\left(c_{2}-c_{1}\right)}{2 s^{2}}\right)^{2} s^{2}\right)\left[\left(\frac{p_{2}+p_{1}-\mathrm{i}\left(c_{2}-c_{1}\right)}{2 s^{2}}\right)^{2}+\frac{1}{2 s^{2}}\right] .
\end{aligned}
$$

Now consider the integration over $c_{1}$. Notice that the modulus of the integrand is dominated by a Gaussian times a polynomial, where the width of the Gaussian is proportional to $s$. So, for small semiclassicality parameter $s$, the main contribution of the integral over $c_{1}$ will come from the point where the gaussian is peaked, that is $-c_{2} \in\left[-\frac{\pi}{\mu_{0}}, \frac{\pi}{\mu_{0}}\right]$. At this point, $\chi\left(-c_{2}\right)=1$. Therefore we have for the integral:

$$
\begin{aligned}
& \frac{\mu_{0}}{\sqrt{\pi s^{6}}} \int_{\mathbb{R}} \frac{\mathrm{d} c_{1}}{2 \pi} \int_{-\frac{\pi}{\mu_{0}}}^{\frac{\pi}{\mu_{0}}} \frac{\mathrm{d} c_{2}}{2 \pi} \int_{\mathbb{R}^{2}} \mathrm{~d} p_{1} \mathrm{~d} p_{2} \exp \left(-\left(v-\frac{p_{1}}{s^{2}}\right)^{2} \frac{s^{2}}{2}\right) \exp \left(-\left(\mu-\frac{p_{2}}{s^{2}}\right)^{2} \frac{s^{2}}{2}\right) \mathrm{e}^{\mathrm{i} v c_{1}} \mathrm{e}^{-\mathrm{i} \mu c_{2}} \\
& \times \exp \left(-\frac{p_{1}^{2}+p_{2}^{2}}{2 s^{2}}\right) \sin ^{8}\left[\mu_{0}\left(c_{1}+c_{2}-\mathrm{i}\left(p_{2}-p_{1}\right)\right)\right] \\
& \times \exp \left(\left(\frac{p_{2}+p_{1}-\mathrm{i}\left(c_{2}-c_{1}\right)}{2 s^{2}}\right)^{2} s^{2}\right)\left[\left(\frac{p_{2}+p_{1}-\mathrm{i}\left(c_{2}-c_{1}\right)}{2 s^{2}}\right)^{2}+\frac{1}{2 s^{2}}\right] \\
& \times\left(1+O\left(s^{\infty}\right)\right)
\end{aligned}
$$


We omit the $\left(1+O\left(s^{\infty}\right)\right)$ in the following calculations. Shifting the integration of $c_{1}$ by $c_{2}$ and rescaling the integration of $c_{2}$ by $\mu_{0}$, we obtain

$$
\begin{aligned}
\frac{1}{\sqrt{\pi s^{6}}} \int_{\mathbb{R}} \frac{\mathrm{d} c_{1}}{2 \pi} & \int_{-\pi}^{\pi} \frac{\mathrm{d} c_{2}}{2 \pi} \int_{\mathbb{R}^{2}} \mathrm{~d} p_{1} \mathrm{~d} p_{2} \exp \left(-\left(v-\frac{p_{1}}{s^{2}}\right)^{2} \frac{s^{2}}{2}\right) \exp \left(-\left(\mu-\frac{p_{2}}{s^{2}}\right)^{2} \frac{s^{2}}{2}\right) \\
& \times \exp \left(\mathrm{i} v c_{1}-\mathrm{i} \frac{\mu-v}{\mu_{0}} c_{2}\right) \exp \left(-\frac{p_{1}^{2}+p_{2}^{2}}{2 s^{2}}\right) \\
& \times \sin ^{8}\left[2 c_{2}+\mu_{0}\left(c_{1}-\mathrm{i}\left(p_{2}-p_{1}\right)\right)\right] \\
& \times \exp \left(-\left(\frac{c_{1}-\mathrm{i}\left(p_{1}+p_{2}\right)}{2 s^{2}}\right)^{2} s^{2}\right)\left[\frac{1}{2 s^{2}}-\left(\frac{c_{1}-\mathrm{i}\left(p_{1}+p_{2}\right)}{2 s^{2}}\right)^{2}\right] .
\end{aligned}
$$

Note that the integrand is analytic in $c_{1}$. We now make a shift of the contour of integration of $c_{2}$ by $\mathrm{i}\left(p_{1}+p_{2}\right)$. When doing this, one has to take care about the convergence of the integral. As one can check, no problem arises in our case. We obtain

$$
\begin{aligned}
\frac{1}{\sqrt{\pi s^{6}}} \int_{\mathbb{R}} \frac{\mathrm{d} c_{1}}{2 \pi} & \int_{-\pi}^{\pi} \frac{\mathrm{d} c_{2}}{2 \pi} \int_{\mathbb{R}^{2}} \mathrm{~d} p_{1} \mathrm{~d} p_{2} \exp \left(-\left(v-\frac{p_{1}}{s^{2}}\right)^{2} \frac{s^{2}}{2}\right) \exp \left(-\left(\mu-\frac{p_{2}}{s^{2}}\right)^{2} \frac{s^{2}}{2}\right) \\
& \times \exp \left(v\left(p_{1}+p_{2}\right)\right) \exp \left(\mathrm{i} v c_{1}-\mathrm{i} \frac{\mu-v}{\mu_{0}} c_{2}\right) \exp \left(-\frac{p_{1}^{2}+p_{2}^{2}}{2 s^{2}}\right) \\
& \times \sin ^{8}\left(2 c_{2}+\mu_{0}\left(c_{1}+2 \mathrm{i} p_{2}\right)\right) \mathrm{e}^{-\frac{c_{1}^{2}}{4 s^{2}}}\left(\frac{1}{2 s^{2}}-\frac{c_{1}^{2}}{4 s^{4}}\right) .
\end{aligned}
$$

It is now convenient to carry out the integration over $c_{2}$. We note that

$$
\int_{-\pi}^{\pi} \frac{\mathrm{d} c_{2}}{2 \pi} \sin ^{8}\left(2 c_{2}+a\right) \mathrm{e}^{\mathrm{i} N c_{2}}=\frac{1}{256}(-1)^{\frac{N}{4}}\left(\begin{array}{c}
8 \\
4+\frac{N}{4}
\end{array}\right) \mathrm{e}^{-\mathrm{i} a \frac{N}{2}},
$$

when $N$ is divisible by four. We get

$$
\begin{aligned}
\frac{1}{256}(-1)^{\frac{v-\mu}{4 \mu_{0}}} \frac{1}{\sqrt{\pi s^{6}}}\left(\begin{array}{c}
8 \\
4+\frac{v-\mu}{4 \mu_{0}}
\end{array}\right) \int_{\mathbb{R}^{3}} \frac{\mathrm{d} c_{1}}{2 \pi} \mathrm{d} p_{1} \mathrm{~d} p_{2} \exp \left(-\left(v-\frac{p_{1}}{s^{2}}\right)^{2} \frac{s^{2}}{2}\right) \\
\quad \times \exp \left(-\left(\mu-\frac{p_{2}}{s^{2}}\right)^{2} \frac{s^{2}}{2}\right) \exp \left(v p_{2}+\mu p_{1}\right) \exp \left(-\frac{p_{1}^{2}+p_{2}^{2}}{2 s^{2}}\right) \\
\quad \times \exp \left(-\mathrm{i} \frac{v+\mu}{2} c_{1}\right) \exp \left(-\frac{c_{1}^{2}}{4 s^{2}}\right)\left(\frac{1}{2 s^{2}}-\frac{c_{1}^{2}}{4 s^{4}}\right) .
\end{aligned}
$$

Integration over $c_{1}$ gives

$$
\begin{aligned}
\frac{1}{256}(-1)^{\frac{v-\mu}{4 \mu_{0}}} & \frac{1}{\pi s^{2}}\left(\begin{array}{c}
8 \\
4+\frac{v-\mu}{4 \mu_{0}}
\end{array}\right) \int_{\mathbb{R}^{2}} \mathrm{~d} p_{1} \mathrm{~d} p_{2} \exp \left(-\left(v-\frac{p_{1}}{s^{2}}\right)^{2} \frac{s^{2}}{2}\right) \exp \left(-\left(\mu-\frac{p_{2}}{s^{2}}\right)^{2} \frac{s^{2}}{2}\right) \\
& \times \exp \left(v p_{2}+\mu p_{1}\right) \exp \left(-\frac{p_{1}^{2}+p_{2}^{2}}{2 s^{2}}\right) \exp \left(-s^{2}\left(\frac{v+\mu}{2}\right)^{2}\right)\left(\frac{v+\mu}{2}\right)^{2} \\
= & \frac{1}{256}(-1)^{\frac{v-\mu}{4 \mu_{0}}}\left(\begin{array}{c}
8 \\
4+\frac{v-\mu}{4 \mu_{0}}
\end{array}\right)\left(\frac{v+\mu}{2}\right)^{2} \mathrm{e}^{v \mu s^{2}}
\end{aligned}
$$

We now reinsert the correction that we have omitted since formula (4.17) and are left with

$$
\frac{1}{256}(-1)^{\frac{v-\mu}{4 \mu_{0}}}\left(\begin{array}{c}
8 \\
4+\frac{v-\mu}{4 \mu_{0}}
\end{array}\right)\left(\frac{v+\mu}{2}\right)^{2} \mathrm{e}^{\nu \mu s^{2}}\left(1+O\left(s^{\infty}\right)\right) .
$$

For all higher orders of the exponentiated derivative in (4.11) we obtain similar but more complicated expressions, that have in common the fact that they stay finite, as $s$ tends to 0 . 
The integration over the term containing $\zeta$ in (4.11) is easily performed and yields, up to terms of order $O\left(s^{2}\right)$ :

$$
\begin{aligned}
& \frac{\mu_{0}^{3}}{16 \sqrt{\pi s^{6}}} \int_{\left[-\frac{\pi}{\mu_{0}}, \frac{\pi}{\mu_{0}}\right]^{2}} \frac{\mathrm{d} c_{1}}{2 \pi} \frac{\mathrm{d} c_{2}}{2 \pi} \int_{\mathbb{R}^{2}} \mathrm{~d} p_{1} \mathrm{~d} p_{2} \exp \left(-\left(v-\frac{p_{1}}{s^{2}}\right)^{2} \frac{s^{2}}{2}\right) \exp \left(-\left(\mu-\frac{p_{2}}{s^{2}}\right)^{2} \frac{s^{2}}{2}\right) \\
& \quad \times\left.\mathrm{e}^{\mathrm{i} v c_{1}} \mathrm{e}^{-\mathrm{i} \mu c_{2}} \exp \left(-\frac{p_{1}^{2}+p_{2}^{2}}{2 s^{2}}\right) \exp \left(s^{2} \frac{\partial^{2}}{\partial z^{2}}\right)\right|_{z=\mathrm{i}(u+\bar{v}) \mu_{0}} \zeta(z) \\
& \quad \times \sum_{n \in \mathbb{Z}} \exp \left(\frac{2 \pi \mathrm{i} n \delta}{\mu_{0}}\right) \exp \left(\left(\frac{p_{2}+p_{1}-\mathrm{i}\left(c_{2}-c_{1}\right)}{2 s^{2}}+\mathrm{i} \frac{\pi n}{\mu_{0} s^{2}}\right)^{2} s^{2}\right) \\
&= \frac{\mu_{0}^{2}}{32}\left[\frac{193}{48} \delta_{\left|\frac{v-\mu}{4 \mu_{0}}\right|, 4}+\frac{109}{6} \delta_{\left|\frac{v-\mu}{4 \mu_{0}}\right|, 3}+\frac{487}{12} \delta_{\left|\frac{v-\mu}{4 \mu_{0}}\right|, 2}+\frac{55}{6} \delta_{\left|\frac{v-\mu}{4 \mu_{0}}\right|, 1}+\frac{35}{48} \delta_{\left|\frac{v-\mu}{4 \mu_{0}}\right|, 0}\right] \\
& \times\left(1+O\left(s^{2}\right)\right) .
\end{aligned}
$$

We now go back to the formula for the resolution of unity in (3.32) and note that the right-hand side does not depend on $s$, so

$$
\begin{aligned}
\operatorname{id}_{\mathcal{H}_{\delta}} & =\lim _{s \rightarrow 0} \operatorname{id}_{\mathcal{H}_{\delta}} \\
& =\lim _{s \rightarrow 0} \frac{\mu_{0}}{\sqrt{\pi s^{2}}} \int_{-\frac{\pi}{\mu_{0}}}^{\frac{\pi}{\mu_{0}}} \frac{\mathrm{d} c}{2 \pi} \int_{\mathbb{R}} \mathrm{d} p \exp \left(-\frac{p^{2}}{s^{2}}\right)|c-\mathrm{i} p\rangle\langle c-\mathrm{i} p| .
\end{aligned}
$$

This allows us to drop all terms of order $O(s)$ in our approximation, and we are left with

$$
\begin{aligned}
&\left\langle v\left|\hat{M}^{2}\right| \mu\right\rangle \approx \frac{1}{256}(-1)^{\frac{v-\mu}{4 \mu_{0}}}\left(\begin{array}{c}
8 \\
4+\frac{v-\mu}{4 \mu_{0}}
\end{array}\right)\left(\frac{v+\mu}{2}\right)^{2}-\frac{\mu_{0}^{2}}{32}(-1)^{\frac{v-\mu}{4 \mu_{0}}}\left[\frac{193}{48} \delta_{\left|\frac{v-\mu}{4 \mu_{0}}\right|, 4}+\frac{109}{6} \delta_{\left|\frac{v-\mu}{4 \mu_{0}}\right|, 3}\right. \\
&\left.+\frac{487}{12} \delta_{\left|\frac{v-\mu}{4 \mu_{0}}\right|, 2}+\frac{55}{6} \delta_{\left|\frac{v-\mu}{4 \mu_{0}}\right|, 1}+\frac{35}{48} \delta_{\left|\frac{v-\mu}{4 \mu_{0}}\right|, 0}\right] .
\end{aligned}
$$

Note that the approximation now comes from neglecting the $R(x)$-term in formula (4.3), but no longer from $s$-corrections, since we have gone over to the limit $s \rightarrow 0$, in which all these terms vanish.

\section{Computation of $\left\langle\nu\left|\hat{M}^{2 n}\right| \mu\right\rangle$ for $n>1$ and rigging map}

\subsection{Expectation values of $\hat{M}^{2 n}$}

The approximate formula for the matrix elements of $\hat{M}^{2}$ has been calculated in the last section. As demanded the approximation vanishes if $\frac{\nu-\mu}{4 \mu_{0}}$ is not an integer. If it is, the matrix elements can be approximated by

$$
\begin{aligned}
\left\langle\nu\left|\hat{M}^{2}\right| \mu\right\rangle \approx & \frac{(-1)^{N}}{256}\left[\left(\begin{array}{c}
8 \\
4+N
\end{array}\right)\left(\frac{v+\mu}{2}\right)^{2}+\frac{193}{6} \mu_{0}^{2} \delta_{|N|, 4}+\frac{436}{3} \mu_{0}^{2} \delta_{|N|, 3}\right. \\
& \left.+\frac{974}{3} \mu_{0}^{2} \delta_{|N|, 2}+\frac{220}{3} \mu_{0}^{2} \delta_{|N|, 1}+\frac{35}{6} \mu_{0}^{2} \delta_{|N|, 0}\right]
\end{aligned}
$$

with $N=\frac{\nu-\mu}{4 \mu_{0}}$. So

$$
\left\langle v\left|\hat{M}^{2}\right| \mu\right\rangle=\frac{(-1)^{N}}{256}\left(\begin{array}{c}
8 \\
4+N
\end{array}\right)\left(\frac{v+\mu}{2}\right)^{2}+R(v, \mu, n),
$$

with $R$ being correction terms that we neglect for the time being. 
Since the $|\nu\rangle$ with $v \in \delta+4 \mu_{0} \mathbb{Z}$ provide a basis of $\mathcal{H}_{\delta}$, we can easily compute the matrix elements of $\hat{M}^{2 n}$ :

$$
\begin{aligned}
&\left\langle\nu\left|\hat{M}^{2 n}\right| \mu\right\rangle \approx \frac{(-1)^{N}}{256^{n}} \sum_{\lambda_{1} \in \delta+4 \mu_{0} \mathbb{Z}}\left(\begin{array}{c}
8 \\
4+\frac{\nu-\lambda_{1}}{4 \mu_{0}}
\end{array}\right)\left(\begin{array}{c}
8 \\
4+\frac{\lambda_{1}-\lambda_{2}}{4 \mu_{0}}
\end{array}\right) \cdots\left(\begin{array}{c}
8 \\
4+\frac{\lambda_{n-1}-\mu}{4 \mu_{0}}
\end{array}\right) \\
& \vdots \\
& \lambda_{n-1} \in \delta+4 \mu_{0} \mathbb{Z} \\
& \times\left(\frac{v+\lambda_{1}}{2}\right)^{2}\left(\frac{\lambda_{1}+\lambda_{2}}{2}\right)^{2} \cdots\left(\frac{\lambda_{n-1}+\mu}{2}\right)^{2} .
\end{aligned}
$$

It is very difficult to give a closed expression for this formula. Still, we want to show a way of calculating these matrix elements in principle. For this, we first rewrite the summation:

$$
\begin{aligned}
\left\langle v\left|\hat{M}^{2 n}\right| \mu\right\rangle \approx \frac{(-1)^{\frac{v-\mu}{4 \mu_{0}}}}{256^{n}} \sum_{\substack{l_{1}, \ldots, \lambda_{n} \in \mathbb{Z} \\
\sum_{k} l_{k}=\frac{v-\mu}{4 \mu_{0}}}} \prod_{k=1}^{n}\left[\left(\begin{array}{c}
8 \\
4+l_{k}
\end{array}\right)\right] \\
\times \prod_{k=1}^{n}\left[\left(\frac{v+\mu}{2}-\mu_{0} \sum_{j=1}^{k-1} l_{j}+\mu_{0} \sum_{j=k+1}^{n} l_{j}\right)^{2}\right] .
\end{aligned}
$$

Note that, whenever $\frac{\nu-\mu}{4 \mu_{0}}$ is not an integer, the sum is empty and thus, by convention, 0 , as demanded. Because of

$$
\begin{aligned}
\left(\frac{v+\mu}{2}-\mu_{0}\right. & \left.\sum_{j=1}^{k-1} l_{j}+\mu_{0} \sum_{j=k+1}^{n} l_{j}\right)^{2}=\frac{\mathrm{d}^{2}}{\left.\mathrm{~d} a_{k}^{2}\right|_{a_{k}=0}} \\
& \times \exp \left(a_{k}\left(\frac{v+\mu}{2}-\mu_{0} \sum_{j=1}^{k-1} l_{j}+\mu_{0} \sum_{j=k+1}^{n} l_{j}\right)\right)
\end{aligned}
$$

and

$$
\delta_{\sum_{k} l_{k}, \frac{v-\mu}{4 \mu_{0}}}=\int_{0}^{2 \pi} \frac{\mathrm{d} b}{2 \pi} \exp \left(\mathrm{i} b\left(\frac{v-\mu}{4 \mu_{0}}-\sum_{k} l_{k}\right)\right),
$$

We can rewrite (5.2) in the following way, after shifting each of the sums over $l_{k}$ by -4 :

$$
\begin{aligned}
\left\langle v\left|\hat{M}^{2 n}\right| \mu\right\rangle & \approx \frac{(-1)^{\frac{v-\mu}{4 \mu_{0}}}}{256^{n}} \sum_{l_{1}, \ldots, l_{n} \in \mathbb{Z}} \int_{0}^{2 \pi} \frac{\mathrm{d} b}{2 \pi} \exp \left(\mathrm{i} b\left(\frac{v-\mu}{4 \mu_{0}}-\sum_{k} l_{k}+4 n\right)\right)\left[\prod_{k=1}^{n}\left(\begin{array}{l}
8 \\
l_{k}
\end{array}\right)\right] \\
& \times \prod_{k=1}^{n}\left[\frac{\mathrm{d}^{2}}{\left.\mathrm{~d} a_{k}^{2}\right|_{a_{k}=0}} \exp \left(a_{k}\left(\frac{v+\mu}{2}-\mu_{0} \sum_{j=1}^{k-1} l_{j}+\mu_{0} \sum_{j=k+1}^{n} l_{j}+4 \mu_{0}(n-2 k+1)\right)\right]\right. \\
= & \frac{(-1)^{\frac{v-\mu}{4 \mu_{0}}}}{256^{n}} \sum_{l_{1}, \ldots, l_{n} \in \mathbb{Z}} \int_{0}^{2 \pi} \frac{\mathrm{d} b}{2 \pi} \exp \left(\mathrm{i} b\left(\frac{v-\mu}{4 \mu_{0}}-\sum_{k} l_{k}+4 n\right)\right)\left[\prod_{k=1}^{n}\left(\begin{array}{l}
8 \\
l_{k}
\end{array}\right)\right] \\
& \times\left[\prod_{k=1}^{n} \frac{\mathrm{d}^{2}}{\left.\mathrm{~d} a_{k}^{2}\right|_{a_{k}=0}}\right] \prod_{k=1}^{n}\left[\frac{\mathrm{d}^{2}}{\mathrm{~d} a_{k}^{2}} \exp \left(4 a_{k} \mu_{0}(n-2 k+1)\right)\right. \\
& \left.\times \exp \left(l_{k} \mu_{0}\left(\sum_{j=1}^{k-1} a_{j}-\sum_{j=k+1}^{n} a_{j}\right)\right)\right]
\end{aligned}
$$


By the binomial formula, this is

$$
\begin{aligned}
\left\langle\nu\left|\hat{M}^{2 n}\right| \mu\right\rangle \approx & \frac{(-1)^{\frac{v-\mu}{4 \mu_{0}}}}{256^{n}} \int_{0}^{2 \pi} \frac{\mathrm{d} b}{2 \pi} \exp \left(\mathrm{i} b\left(\frac{\nu-\mu}{4 \mu_{0}}+4 n\right)\right) \\
& \times\left[\prod_{k=1}^{n} \frac{\mathrm{d}^{2}}{\mathrm{~d} a_{k \mid a_{k}}^{2}=0}\right] \prod_{k=1}^{n} \exp \left(4 a_{k} \mu_{0}(n-2 k+1)\right)\left(1+\zeta_{k}\left(a_{1}, \ldots, a_{n}, b\right)\right)^{8}
\end{aligned}
$$

with

$$
\zeta_{k}\left(a_{1}, \ldots, a_{n}, b\right)=\exp \left(\mu_{0}\left(\sum_{j=1}^{k-1} a_{j}-\sum_{j=k+1}^{n} a_{j}\right)-\mathrm{i} b\right) .
$$

It is now straightforward, though tedious, to perform the $2 n$ differentiations. Since

$$
\frac{\mathrm{d}}{\mathrm{d} a_{k}}\left(1+\zeta_{l}(\vec{a}, b)\right)^{n}=n \operatorname{sgn}(1-\mathrm{k})\left(\left(1+\zeta_{1}(\vec{a}, \mathrm{~b})\right)^{\mathrm{n}}-\left(1+\zeta_{1}(\vec{a}, \mathrm{~b})\right)^{\mathrm{n}-1}\right),
$$

it is predictable that the differentiations and setting of $\vec{a}=0$ afterwards will yield the result

$$
\begin{gathered}
{\left[\prod_{k=1}^{n} \frac{\mathrm{d}^{2}}{\left.\mathrm{~d} a_{k}^{2}\right|_{a_{k}=0}}\right] \prod_{k=1}^{n} \exp \left(4 a_{k} \mu_{0}(n-2 k+1)\right)\left(1+\zeta_{k}\left(a_{1}, \ldots, a_{n}, b\right)\right)^{8}} \\
=\sum_{m=0}^{8 n} P_{n, m}(v, \mu)\left(1+\mathrm{e}^{-\mathrm{i} b}\right)^{8 n-m} \mathrm{e}^{\mathrm{i} b m},
\end{gathered}
$$

where the $P_{n, m}(\nu, \mu)$ are polynomials in $v$ and $\mu$. Since

$\int_{0}^{2 \pi} \frac{\mathrm{d} b}{2 \pi} \exp \left(\mathrm{i} b\left(\frac{v-\mu}{4 \mu_{0}}+4 n\right)\right)\left(1+\mathrm{e}^{-\mathrm{i} b}\right)^{8 n-m} \mathrm{e}^{\mathrm{i} b m}=\left(\begin{array}{c}8 n-m \\ 4 n+\frac{\nu-\mu}{4 \mu_{0}}-m\end{array}\right)$

and

$$
\begin{array}{r}
\left(\begin{array}{c}
8 n-m \\
4 n+\frac{\nu-\mu}{4 \mu_{0}}-m
\end{array}\right)=\left(\frac{4 n+\frac{\nu-\mu}{4 \mu_{0}}}{8 n}\right)\left(\frac{4 n+\frac{\nu-\mu}{4 \mu_{0}}-1}{8 n-1}\right) \cdots \\
\cdots\left(\frac{4 n+\frac{\nu-\mu}{4 \mu_{0}}-m+1}{8 n-m+1}\right)\left(\begin{array}{c}
8 n \\
4 n+\frac{\nu-\mu}{4 \mu_{0}}
\end{array}\right),
\end{array}
$$

the form of the result is known:

$$
\left\langle\nu\left|\hat{M}^{2 n}\right| \mu\right\rangle \approx \frac{(-1)^{\frac{\nu-\mu}{4 \mu_{0}}}}{256^{n}} Q_{n}(\nu, \mu)\left(\begin{array}{c}
8 n \\
4 n+\frac{\nu-\mu}{4 \mu_{0}}
\end{array}\right),
$$

where $Q_{n}(v, \mu)$ is another polynomial in $v$ and $\mu$, consisting of linear combinations of the $P_{n, m}(\nu, \mu)$. However, it is a considerable computational task to obtain the polynomials $P_{n, m}(\nu, \mu)$ for higher $m$, and, to the best of the authors' knowledge, no closed formula for them exists. So, although one can compute the approximate matrix elements of $\left\langle\nu\left|\hat{M}^{2 n}\right| \mu\right\rangle$ explicitly order by order, no closed formula for them is known.

\subsection{Further approximations}

In order to compute the rigging map approximatively, we start again from formula (5.2)

$$
\left\langle v\left|\hat{M}^{2 n}\right| \mu\right\rangle \approx \frac{(-1)^{\frac{v-\mu}{4 \mu_{0}}}}{256^{n}} \sum_{\substack{l_{1}, \ldots, l_{n} \in \mathbb{Z} \\
\sum_{k} l_{k}=\frac{v-\mu}{4 \mu_{0}}}} \prod_{k=1}^{n}\left[\left(\begin{array}{c}
8 \\
4+l_{k}
\end{array}\right)\right] \prod_{k=1}^{n}\left[\left(\frac{v+\mu}{2}-\Delta_{k}(\vec{l})\right)^{2}\right]
$$


with

$$
\Delta_{k}(\vec{l}):=\mu_{0} \sum_{j=1}^{k-1} l_{j}-\mu_{0} \sum_{j=k+1}^{n} l_{j}
$$

Now assume that $v-\mu$ is small compared to $v+\mu$. Then the summation of the $l_{k}$ ranges over $-4, \ldots, 4$, and since the binomial prefactors are nearly Gaussian distributions in the $l_{k}$, it is safe to assume that the largest contribution to the sum will be made by products, where all the $l_{k}$ are close to 0 , compared to 4 , hence the $\Delta_{k}(\vec{l})$ are much smaller than $\frac{\nu+\mu}{2}$. So, as a test, we could expand formula (5.13) into powers of the sums over the $\Delta_{k}(\vec{l})$ :

$$
\begin{aligned}
\left\langle\nu\left|\hat{M}^{2 n}\right| \mu\right\rangle \approx & \frac{(-1)^{\frac{\nu-\mu}{4 \mu_{0}}}}{256^{n}} \sum_{\substack{l_{1}, \ldots, l_{n} \in \mathbb{Z} \\
\sum_{k} l_{k}=\frac{\nu-\mu}{4 \mu_{0}}}} \prod_{k=1}^{n}\left[\left(\begin{array}{c}
8 \\
4+l_{k}
\end{array}\right)\right]\left[\left(\frac{\nu+\mu}{2}\right)^{2 n}-2 \sum_{k=1}^{n} \Delta_{k}(\vec{l})\left(\frac{\nu+\mu}{2}\right)^{2 n-1}\right. \\
& \left.+\left(2\left(\sum_{k=1}^{n} \Delta_{k}(\vec{l})\right)^{2}-\sum_{k=1}^{n} \Delta_{k}(\vec{l})^{2}\right)\left(\frac{v+\mu}{2}\right)^{2 n-2}+\ldots\right]
\end{aligned}
$$

For what follows, we assume $n>1$, since only then are the $\Delta_{k}(\vec{l}) \neq 0$. The cases $n=0$ and $n=1$ are trivial and already known respectively.

Now first we observe that

$$
\sum_{\substack{l_{1}, \ldots, l_{n} \in \mathbb{Z} \\ \sum_{k} l_{k}=\frac{\nu-\mu}{4 \mu_{0}}}} f(\vec{l})=\sum_{\substack{l_{1}, \ldots, l_{n} \in \mathbb{Z} \\ \sum_{k} l_{k}=\frac{\nu-\mu}{4 \mu_{0}}}} \frac{1}{n !} \sum_{\sigma \in \operatorname{Sym}_{\mathrm{n}}} f(\sigma(\vec{l})),
$$

where $\operatorname{Sym}_{\mathrm{n}}$ is the permutation group in $n$ elements and $\sigma(\vec{l})$ means the vector, of which the $n$ entries in the vector $\vec{l}$ are permuted according to $\sigma$. Our second observation is that

$$
\sum_{\sigma \in \operatorname{Sym}_{\mathrm{n}}} \sum_{k=1}^{n} \Delta_{k}(\sigma(\vec{l}))=0,
$$

so the 'linear' term in (5.15) vanishes, and, keeping only the first two terms of our expansion, we are left with

$$
\begin{aligned}
& \left\langle\nu\left|\hat{M}^{2 n}\right| \mu\right\rangle \approx \frac{(-1)^{\frac{v-\mu}{4 \mu_{0}}}}{256^{n}} \sum_{\substack{l_{1}, \ldots, l_{n} \in \mathbb{Z} \\
\sum_{k} l_{k}=\frac{\nu-\mu}{4 \mu_{0}}}} \prod_{k=1}^{n}\left[\left(\begin{array}{c}
8 \\
4+l_{k}
\end{array}\right)\right] \\
& \times\left[\left(\frac{v+\mu}{2}\right)^{2 n}+\left(2\left(\sum_{k=1}^{n} \Delta_{k}(\vec{l})\right)^{2}-\sum_{k=1}^{n} \Delta_{k}(\vec{l})^{2}\right)\left(\frac{v+\mu}{2}\right)^{2 n-2}\right] \\
& =\frac{(-1)^{\frac{\nu-\mu}{4 \mu_{0}}}}{256^{n}}\left(\begin{array}{c}
8 n \\
4 n+\frac{\nu-\mu}{4 \mu_{0}}
\end{array}\right)\left(\frac{\nu+\mu}{2}\right)^{2 n}+\frac{(-1)^{\frac{\nu-\mu}{4 \mu_{0}}}}{256^{n} n !}\left(\frac{\nu+\mu}{2}\right)^{2 n-2} \\
& \times\left[2 \sum_{\substack{l_{1}, \ldots, l_{n} \in \mathbb{Z} \\
\sum_{k} l_{k}=\frac{v-\mu}{4 \mu_{0}}}} \prod_{k=1}^{n}\left[\left(\begin{array}{c}
8 \\
4+l_{k}
\end{array}\right)\right] \sum_{\sigma \in \operatorname{Sym}_{\mathrm{n}}}\left(\sum_{k=1}^{n} \Delta_{k}(\sigma(\vec{l}))\right)^{2}\right.
\end{aligned}
$$




$$
\left.-\sum_{\substack{l_{1}, \ldots, l_{n} \in \mathbb{Z} \\
\sum_{k} l_{k}=\frac{v-\mu}{4 \mu_{0}}}} \prod_{k=1}^{n}\left[\left(\begin{array}{c}
8 \\
4+l_{k}
\end{array}\right)\right] \sum_{\sigma \in \operatorname{Sym}_{\mathrm{n}}} \sum_{k=1}^{n} \Delta_{k}(\sigma(\vec{l}))^{2}\right] .
$$

Let us consider the middle term in formula (5.19). First we note that $\frac{1}{\mu_{0}} \sum_{k=1}^{n} \Delta_{k}(\vec{l})= \begin{cases}(n-1)\left(l_{n}-l_{1}\right)+(n-3)\left(l_{n-1}-l_{2}\right)+\cdots+2\left(l_{\frac{n+3}{2}}-l_{\frac{n-1}{2}}\right) & n \text { odd } \\ (n-1)\left(l_{n}-l_{1}\right)+(n-3)\left(l_{n-1}-l_{2}\right)+\cdots+\left(l_{\frac{n}{2}+1}-l_{\frac{n}{2}}\right) & n \text { even. }\end{cases}$

So, squaring this, one obtains a sum over two different types of terms, in particular

$$
\left(l_{a}-l_{b}\right)^{2} \quad \text { and } \quad\left(l_{a}-l_{b}\right)\left(l_{c}-l_{d}\right),
$$

where in the first term $a$ and $b$ are different and in the second term all $a, b, c d$ are different. Just because of this, terms of the last kind cancel when summing over $\operatorname{Sym}_{\mathrm{n}}$ :

$$
\sum_{\sigma \in \operatorname{Sym}_{\mathrm{n}}}\left(l_{\sigma(a)}-l_{\sigma(b)}\right)\left(l_{\sigma(c)}-l_{\sigma(d)}\right)=0 .
$$

So we can only keep the terms of the first kind and, with (5.20), we obtain:

$$
\begin{aligned}
\sum_{\sigma \in \operatorname{Sym}_{\mathrm{n}}}\left(\sum_{k=1}^{n} \Delta_{k}(\sigma(\vec{l}))\right)^{2} & =\mu_{0}^{2} \sum_{\sigma \in \operatorname{Sym}_{\mathrm{n}}}\left((n-1)^{2}\left(l_{\sigma(n)}-l_{\sigma(1)}\right)^{2}\right. \\
& \left.+(n-3)^{2}\left(l_{\sigma(n-1)}-l_{\sigma(2)}\right)^{2}+\cdots\right) \\
& =2 k_{n} \mu_{0}^{2}(n-2) ! \sum_{k=1}^{n} \sum_{m=1}^{n}\left(l_{k}-l_{m}\right)^{2},
\end{aligned}
$$

where

$$
k_{n}= \begin{cases}1^{2}+3^{2}+\cdots+(n-1)^{2} & n \text { even } \\ 2^{2}+4^{2}+\cdots+(n-1)^{2} & n \text { odd }\end{cases}
$$

A short analysis reveals that $k_{n}=\frac{1}{6}(n-1) n(n+1)$, independently of whether $n$ is even or odd. So, combining the results from (5.19) and (5.23), we get

$$
\begin{aligned}
\left\langle v\left|\hat{M}^{2 n}\right| \mu\right\rangle \approx & \frac{(-1)^{\frac{v-\mu}{4 \mu_{0}}}}{256^{n}}\left(\begin{array}{c}
8 n \\
4 n+\frac{v-\mu}{4 \mu_{0}}
\end{array}\right)\left(\frac{v+\mu}{2}\right)^{2 n}+2 \mu_{0}^{2} \frac{(-1)^{\frac{\nu-\mu}{4 \mu_{0}}}}{256^{n}} \frac{n+1}{3}\left(\frac{v+\mu}{2}\right)^{2 n-2} \\
& \times \sum_{\substack{l_{1}, \ldots, l_{n} \in \mathbb{Z} \\
\sum_{k} l_{k}=\frac{\nu-\mu}{4 \mu_{0}}}}\left[\prod_{k=1}^{n}\left(\begin{array}{c}
8 \\
4+l_{k}
\end{array}\right)\right] \sum_{k, m}\left(l_{k}-l_{m}\right)^{2}-\frac{(-1)^{\frac{\nu-\mu}{4 \mu_{0}}}}{256^{n} n !}\left(\frac{v+\mu}{2}\right)^{2 n-2} \\
& \times \sum_{\substack{l_{1}, \ldots, l_{n} \in \mathbb{Z} \\
\sum_{k} l_{k}=\frac{v-\mu}{4 \mu_{0}}}}\left[\prod_{k=1}^{n}\left(\begin{array}{c}
8 \\
4+l_{k}
\end{array}\right)\right] \sum_{\sigma \in \operatorname{Sym}_{\mathrm{n}}} \sum_{k=1}^{n} \Delta_{k}(\sigma(\vec{l}))^{2} .
\end{aligned}
$$

Now

$$
\sum_{k, m}\left(l_{k}-l_{m}\right)^{2}=n \sum_{k=1}^{n} l_{k}^{2}-\left(\sum_{k=1}^{n} l_{k}\right)^{2}
$$


and thus

$$
\begin{aligned}
\left\langle\nu\left|\hat{M}^{2 n}\right| \mu\right\rangle \approx & \frac{(-1)^{\frac{\nu-\mu}{4 \mu_{0}}}}{256^{n}}\left(\begin{array}{c}
8 n \\
4 n+\frac{v-\mu}{4 \mu_{0}}
\end{array}\right)\left(\frac{v+\mu}{2}\right)^{2 n} \\
& -2 \frac{(-1)^{\frac{\nu-\mu}{4 \mu_{0}}}}{256^{n}} \frac{n+1}{3}\left(\begin{array}{c}
8 n \\
4 n+\frac{v-\mu}{4 \mu_{0}}
\end{array}\right)\left(\frac{v+\mu}{2}\right)^{2 n-2}\left(\frac{v-\mu}{4}\right)^{2} \\
& +2 \mu_{0}^{2} \frac{(-1)^{\frac{\nu-\mu}{4 \mu_{0}}}}{256^{n}} \frac{n(n+1)}{3}\left(\frac{v+\mu}{2}\right)^{2 n-2} \sum_{l_{1}, \ldots, l_{n} \in \mathbb{Z}}\left[\prod_{k=1}^{n}\left(\begin{array}{c}
8 \\
4+l_{k}
\end{array}\right)\right] \sum_{k=1}^{n} l_{k}^{2} \\
& -\frac{(-1)^{\frac{v-\mu}{4 \mu_{0}}}}{256^{n} n !}\left(\frac{\nu+\mu}{2}\right)^{2 n-2} \sum_{k}\left[\begin{array}{c}
n \\
l_{1}, \ldots, l_{n} \in \mathbb{Z} \\
\sum_{k} l_{k}=\frac{v-\mu}{4 \mu_{0}}
\end{array}\right.
\end{aligned}
$$

Let us now evaluate the last line in this expression. With a bit of algebra one can show (for $n>1$ ):

$$
\frac{1}{\mu_{0}^{2} n !} \sum_{\sigma \in \operatorname{Sym}_{\mathrm{n}}} \sum_{k=1}^{n} \Delta_{k}(\sigma(\vec{l}))^{2}=(n-1)\left(\sum_{k=1}^{n} l_{k}^{2}\right)+\frac{2}{n !} \frac{(n+1)(n-1)^{2}}{6}\left(\sum_{k<j} l_{k} l_{j}\right),
$$

of which we only keep the first term, because the second one is small compared to it for large $n$.

$$
\begin{aligned}
\left\langle\nu\left|\hat{M}^{2 n}\right| \mu\right\rangle \approx & \frac{(-1)^{\frac{v-\mu}{4 \mu_{0}}}}{256^{n}}\left(\begin{array}{c}
8 n \\
4 n+\frac{v-\mu}{4 \mu_{0}}
\end{array}\right)\left(\frac{v+\mu}{2}\right)^{2 n} \\
& -2 \frac{(-1)^{\frac{v-\mu}{4 \mu_{0}}}}{256^{n}} \frac{n+1}{3}\left(\begin{array}{c}
8 n \\
4 n+\frac{v-\mu}{4 \mu_{0}}
\end{array}\right)\left(\frac{v+\mu}{2}\right)^{2 n-2}\left(\frac{v-\mu}{4}\right)^{2} \\
& +\mu_{0}^{2} \frac{(-1)^{\frac{v-\mu}{4 \mu_{0}}}}{256^{n}} \frac{2 n^{2}-n+3}{3}\left(\frac{v+\mu}{2}\right)^{2 n-2} \sum_{\substack{l_{1}, \ldots, l_{n} \in \mathbb{Z} \\
\sum_{k} l_{k}=\frac{v-\mu}{4 \mu_{0}}}}\left[\prod_{k=1}^{n}\left(\begin{array}{c}
8 \\
4+l_{k}
\end{array}\right)\right] \sum_{k=1}^{n} l_{k}^{2} .
\end{aligned}
$$

The constrained sum can be evaluated by means of the same trick that has been applied to calculate the constrained sum (5.2). Without showing all the steps again, one obtains

$$
\begin{aligned}
\sum_{\substack{l_{1}, \ldots, l_{n} \in \mathbb{Z} \\
\sum_{k} l_{k}=\frac{\nu-\mu}{4 \mu_{0}}}}\left[\prod_{k=1}^{n}\left(\begin{array}{c}
8 \\
4+l_{k}
\end{array}\right)\right] \sum_{k=1}^{n} l_{k}^{2}=n \sum_{\substack{l_{1}, \ldots, l_{n} \in \mathbb{Z} \\
\sum_{k} l_{k}=\frac{\nu-\mu}{4 \mu_{0}}}}\left[\prod_{k=1}^{n}\left(\begin{array}{c}
8 \\
4+l_{k}
\end{array}\right)\right] l_{1}^{2} \\
=n \frac{\mathrm{d}^{2}}{\mathrm{~d} a^{2}} \int_{\mid a=0}^{2 \pi} \frac{\mathrm{d} b}{2 \pi} \mathrm{e}^{-4 a}\left(1+\mathrm{e}^{a+i b}\right)^{8}\left(1+\mathrm{e}^{\mathrm{i} b}\right)^{8(n-1)} \mathrm{e}^{-\mathrm{i} b \frac{v-\mu}{4 \mu_{0}}} \\
=n\left(16\left(\begin{array}{c}
8 n \\
4 n+\frac{v-\mu}{4 \mu_{0}}
\end{array}\right)-56\left(\begin{array}{c}
8 n-1 \\
4 n-1+\frac{v-\mu}{4 \mu_{0}}
\end{array}\right)+56\left(\begin{array}{c}
8 n-2 \\
4 n-2+\frac{v-\mu}{4 \mu_{0}}
\end{array}\right)\right) .
\end{aligned}
$$


With formula (5.11) one can calculate

$$
\begin{gathered}
16\left(\begin{array}{c}
8 n \\
4 n+\frac{v-\mu}{4 \mu_{0}}
\end{array}\right)-56\left(\begin{array}{c}
8 n-1 \\
4 n-1+\frac{\nu-\mu}{4 \mu_{0}}
\end{array}\right)+56\left(\begin{array}{c}
8 n-2 \\
4 n-2+\frac{v-\mu}{4 \mu_{0}}
\end{array}\right) \\
=\left(16-\frac{112 n}{8 n-1}+\frac{7}{(8 n-1) n}\left(\frac{v-\mu}{4 \mu_{0}}\right)^{2}\right)\left(\begin{array}{c}
8 n \\
4 n+\frac{v-\mu}{4 \mu_{0}}
\end{array}\right) .
\end{gathered}
$$

Inserting (5.30) and (5.31) into (5.27) gives

$$
\begin{aligned}
\left\langle\nu\left|\hat{M}^{2 n}\right| \mu\right\rangle \approx & \frac{(-1)^{\frac{v-\mu}{4 \mu_{0}}}}{256^{n}}\left(\begin{array}{c}
8 n \\
4 n+\frac{v-\mu}{4 \mu_{0}}
\end{array}\right)\left(\frac{v+\mu}{2}\right)^{2 n} \\
& +\frac{(-1)^{\frac{v-\mu}{4 \mu_{0}}}}{256^{n}}\left(\begin{array}{c}
8 n \\
4 n+\frac{v-\mu}{4 \mu_{0}}
\end{array}\right) \mu_{0}^{2}\left(\frac{v+\mu}{2}\right)^{2 n-2}\left(\frac{n(n-1)}{8 n-1} \frac{2 n^{2}-n+3}{3}\right) \\
& +\frac{(-1)^{\frac{v-\mu}{4 \mu_{0}}}}{256^{n}}\left(\begin{array}{c}
8 n \\
4 n+\frac{v-\mu}{4 \mu_{0}}
\end{array}\right) \mu_{0}^{2}\left(\frac{v+\mu}{2}\right)^{2 n-2}\left(\frac{v-\mu}{4 \mu_{0}}\right)^{2} \\
& \times \frac{1}{3} \frac{n-1}{8 n-1}\left(16 n^{2}+16 n+21\right) .
\end{aligned}
$$

In our calculations we have assumed $n>1$, but formula (5.32) also produces the right result for $n=1$, as one can readily see. Note that the binomial coefficient for $n=0$ produces a 0 for $v \neq \mu$. For $v=\mu$ and $n=0$ the binomial coefficient gives a 1 , as does the whole expression (5.32). So for $n=0$ the expression (5.32) equals $\delta_{v \mu}$, as expected. Hence, the approximation formula is valid even for all $n \geqslant 0$.

\subsection{The physical inner product}

We see that, considering $v-\mu$ being small compared to $v+\mu$, the first term in (5.32) is large compared to the second and the third, although not uniformly for all $n$. Since to compute the rigging map one has to sum over all the orders of $n$, the contribution of the two correction terms could be considerable if $v+\mu$ is too small. So we see that for small $v+\mu$, the major contribution might not come from the zeroth order term, but from the corrections. Fortunately, one can calculate the corrections to the rigging map order by order, which we will do now. The zeroth order term is

$$
\left\langle\nu\left|\hat{M}^{2 n}\right| \mu\right\rangle \approx \frac{(-1)^{\frac{\nu-\mu}{4 \mu_{0}}}}{256^{n}}\left(\begin{array}{c}
8 n \\
4 n+\frac{\nu-\mu}{4 \mu_{0}}
\end{array}\right)\left(\frac{\nu+\mu}{2}\right)^{2 n} .
$$

The rigging map is given by :

$$
\eta[|v\rangle]|\mu\rangle=\lim _{t \rightarrow 0} \frac{\sum_{n=0}^{\infty} \frac{(-1)^{n}}{n ! t^{n}}\left\langle\nu\left|\hat{M}^{2 n}\right| \mu\right\rangle}{\sum_{n=0}^{\infty} \frac{(-1)^{n}}{n ! t^{n}}\left\langle v_{0}\left|\hat{M}^{2 n}\right| v_{0}\right\rangle},
$$

where $\left|v_{0}\right\rangle$ is a reference vector. With the abbreviations

$$
a=\left|\frac{v+\mu}{32 \mu_{0} \sqrt{t}}\right|, \quad N=\frac{v-\mu}{4 \mu_{0}} .
$$

we can make use of the integral (3.19)

$$
\begin{aligned}
\sum_{n=0}^{\infty} \frac{(-1)^{n}}{2 n !} a^{2 n} & \frac{\Gamma(8 n+1)}{\Gamma(4 n+1+N) \Gamma(4 n+1-N)} \\
& =\frac{1}{2 \pi \mathrm{i}} \int_{-\mathrm{i} \infty-\varepsilon}^{\mathrm{i} \infty-\varepsilon} \mathrm{d} s \Gamma(-s) a^{2 s} \frac{\Gamma(8 s+1)}{\Gamma(4 s+1+N) \Gamma(4 s+1-N)},
\end{aligned}
$$


where the integration contour is chosen to pass slightly left from the pole at 0 . We now apply a coordinate transformation $8 s+1=-r$, so we get

$$
\begin{aligned}
\frac{1}{2 \pi \mathrm{i}} \int_{-\mathrm{i} \infty-\varepsilon}^{\mathrm{i} \infty-\varepsilon} \mathrm{d} s & \Gamma(-s) a^{2 s} \frac{\Gamma(8 s+1)}{\Gamma(4 s+1+N) \Gamma(4 s+1-N)} \\
& =-\frac{1}{16 \pi \mathrm{i}} \int_{\mathrm{i} \infty-1+\varepsilon}^{-\mathrm{i} \infty-1+\varepsilon} \mathrm{d} r \Gamma(-r) a^{-\frac{r+1}{4}} \frac{\Gamma\left(\frac{r+1}{8}\right)}{\Gamma\left(\frac{1}{2}-\frac{r}{8}+N\right) \Gamma\left(\frac{1}{2}-\frac{r}{8}-N\right)} .
\end{aligned}
$$

Observing that the integrand has no poles in $[-1+\varepsilon,-\varepsilon] \times \mathrm{i} \mathbb{R}$, we can shift the integration curve and obtain

$$
\begin{aligned}
\frac{1}{16 \pi \mathrm{i}} \int_{-\mathrm{i} \infty-\varepsilon}^{\mathrm{i} \infty-\varepsilon} \mathrm{d} r \Gamma(-r) a^{-\frac{r+1}{4}} \frac{\Gamma\left(\frac{r+1}{8}\right)}{\Gamma\left(\frac{1}{2}-\frac{r}{8}+N\right) \Gamma\left(\frac{1}{2}-\frac{r}{8}-N\right)} \\
=\frac{1}{8} a^{-\frac{1}{4}} \sum_{n=0}^{\infty} \frac{(-1)^{n}}{n !} a^{-\frac{n}{4}} \frac{\Gamma\left(\frac{n+1}{8}\right)}{\Gamma\left(\frac{1}{2}-\frac{n}{8}+N\right) \Gamma\left(\frac{1}{2}-\frac{n}{8}-N\right)} .
\end{aligned}
$$

This means that, reinserting $a$ and $N$ from (5.35), only the $n=0$-term survives in the limit

$$
\begin{gathered}
\lim _{t \rightarrow 0} t^{-\frac{1}{8}} \sum_{n=0}^{\infty} \frac{(-1)^{n}}{n !}\left(\frac{v+\mu}{32 \mu_{0} t}\right)^{2 n} \frac{\Gamma(8 n+1)}{\Gamma\left(4 n+1+\frac{v-\mu}{4 \mu_{0}}\right) \Gamma\left(4 n+1-\frac{v-\mu}{4 \mu_{0}}\right)} \\
=\frac{1}{8}\left|\frac{32 \mu_{0}}{v+\mu}\right|^{\frac{1}{4}} \frac{\Gamma\left(\frac{1}{8}\right)}{\Gamma\left(\frac{1}{2}+\frac{v-\mu}{4 \mu_{0}}\right) \Gamma\left(\frac{1}{2}-\frac{v-\mu}{4 \mu_{0}}\right)} .
\end{gathered}
$$

Together with

$$
\frac{1}{\Gamma\left(\frac{1}{2}+\frac{\nu-\mu}{4 \mu_{0}}\right) \Gamma\left(\frac{1}{2}-\frac{\nu-\mu}{4 \mu_{0}}\right)}=\frac{1}{\pi}(-1)^{\frac{v-\mu}{4 \mu_{0}}}
$$

and (5.34) we arrive at the result

$$
\eta[|v\rangle]|\mu\rangle \approx\left|\frac{2 v_{0}}{v+\mu}\right|^{\frac{1}{4}}
$$

The above calculation can be carried through likewise with the correction terms in (5.32), which yields, after some careful analysis:

$$
\begin{aligned}
\eta[|v\rangle]|\mu\rangle \approx \mid & \left.\frac{2 v_{0}}{v+\mu}\right|^{\frac{1}{4}}+\left|\frac{2 \mu_{0}}{v+\mu}\right|^{\frac{9}{4}}\left(\frac{\nu_{0}}{\mu_{0}}\right)^{\left(\frac{1}{4}\right)}\left(\frac{231}{64}+\frac{303}{256}\left(\frac{v-\mu}{4 \mu_{0}}\right)^{2}\right) \\
& -\left|\frac{2 \mu_{0}}{v+\mu}\right|^{\frac{9}{4}}\left(\frac{\nu_{0}}{\mu_{0}}\right)^{\left(\frac{1}{4}\right)} \frac{\Gamma\left(\frac{7}{8}\right)^{2}}{\pi \csc \left(\frac{\pi}{8}\right)} \frac{1}{\frac{1}{4}-\left(\frac{v-\mu}{4 \mu_{0}}\right)^{2}}\left(\frac{217}{4}-\frac{217}{16}\left(\frac{v-\mu}{4 \mu_{0}}\right)^{2}\right) .
\end{aligned}
$$

For the calculations with the corrections, the choice of $v_{0}$ results in a choice of normalization of the rigging map. However, the dependence of the normalization on $v_{0}$ is complicated. In (5.43) a particular normalization has been chosen to make the formula comparable to (5.42). 


\subsection{Discussion}

First note that the choice of the reference vector $\left|v_{0}\right\rangle$ is only a choice of normalization of our rigging map. It does not even have to be from the same sector $\delta \in\left[0,4 \mu_{0}\right)$ than $v$ and $\mu$. Note further that in our approximation (5.15) we assumed that $v+\mu$ is large compared to $v-\mu$. In particular, the zeroth order approximation (5.42) cannot be trusted if this condition is violated and, as one can see, it even diverges if $\mu$ approaches $-v$. We will now discuss this formula for the sectors $\delta \neq 0$ and $\delta=0$ separately.

$$
\delta \in\left(0,4 \mu_{0}\right):
$$

The rigging map we have produced suggests, as a zeroth order approximation, that

$$
\eta[|v\rangle] \approx \sum_{\lambda \in \nu+4 \mu_{0} \mathbb{Z}} \frac{1}{|\lambda+v|^{\frac{1}{4}}}\langle\lambda|,
$$

where the result should only be trusted where $\lambda \approx \nu$, and both $\lambda$ and $v$ are large.

We know that by construction (2.19), $\eta[|v\rangle]$ solves the master constraint, i.e.

$$
\eta[|\nu\rangle] \circ \hat{M}=0 .
$$

In section 3.1 we calculated the solutions to the constraint $\hat{C}$ explicitly. There we saw that in each sector $\delta \in\left(0,4 \mu_{0}\right)$ there are two solutions (3.25), one of which is bounded and one of which is unbounded. For all sectors $\delta \neq 0$, the unbounded solution goes like $|\lambda|^{\frac{1}{2}}$, whereas the bounded one goes like $|\lambda|^{-\frac{1}{2}}$ for $|\lambda| \rightarrow \infty$. Furthermore, the master constraint $\hat{M}$ has two further solutions in each sector, given by (3.30), which behave asymptotically like $|\lambda|^{\frac{3}{2}}$ and $|\lambda|^{\frac{5}{2}}$, respectively.

For fixed $|\nu\rangle$, the approximation stays bounded in the regime where it is supposed to be a good approximation to the real $\eta[|v\rangle]$, and goes asymptotically like $|\lambda|^{-\frac{1}{4}}$. Although this is not quite the right asymptotical behaviour, it is safe to assume that the true $\eta[|v\rangle]$ is the bounded solution

$$
\eta[|\nu\rangle]=\sum_{\lambda \in \delta+4 \mu_{0} \mathbb{Z}} \frac{1}{\left|\lambda+\mu_{0}\right|^{\frac{3}{2}}-\left|\lambda-\mu_{0}\right|^{\frac{3}{2}}}\langle\lambda|,
$$

i.e. the one going like $|\lambda|^{-\frac{1}{2}}$. So, although approximation (5.44) does not recover the exact asymptotical behaviour of the physical solution (5.46), it is good enough to identify the right solution (5.46) among the four possible ones (3.25) and (3.30) in each sector $\delta \in\left(0,4 \mu_{0}\right)$.

Numerical comparison of the different approximations (5.2) and (5.32) with the real values for $\left\langle\nu\left|\hat{M}^{2 n}\right| \mu\right\rangle$ for different $n$ indicates that the approximation provided solely by the coherent states (5.2) is quite good, whereas (5.32) is not quite that accurate. This shows that the reason for the slightly different asymptotic behaviour of the final approximation of the physical solution compared to the one expected is caused by further simplifying the formula in section 5.2, in particular by going over from (5.13) to (5.18). This was necessary in order to bring the expressions into a form such that further calculations were possible, but it happened at the cost of accuracy. Still, the approximation formula can in principle be improved by computing and adding further corrections, as happened with the first order corrections in $(5.43)$.

Note that in particular, although the rigging map was defined by the direct integral decomposition of $\hat{M}(2.19)$, the physical Hilbert space contains only solutions of $\hat{M}$ that are also solutions of $\hat{C}$. This shows that, although one had replaced $\hat{C}$ by $\hat{M}$, no information is lost, and hence the master constraint programme proved to be the right tool to define the physical Hilbert space in the presence of a non-self-adjoint constraint. 
Note further that since, analogously to the case of section 3.2, in each sector $\delta \in\left(0,4 \mu_{0}\right)$ there is exactly one solution of nonzero physical norm (hence all other solutions in the same sector are spurious), the Hilbert space is again non-separable, in particular it contains

$$
\bigoplus_{\delta \in\left(0,4 \mu_{0}\right)} \mathbb{C} \subset \mathcal{H}_{\text {phys }}
$$

Hence, in the sectors $\delta \in\left(0,4 \mu_{0}\right)$, the result for the physical Hilbert space provided by the master constraint is the same as for the simplified self-adjoint constraint (3.16), which in fact is classically equivalent to (3.23) apart from $p=0$. So the more complicated, non-self-adjoint constraint (3.23), together with the master constraint leads to the same physical Hilbert space as the simplified constraint (3.16).

$$
\delta=0 \text { : }
$$

The case of $\delta=0$ has to be treated differently than the case $\delta \in\left(0,4 \mu_{0}\right)$. The reason for this is that the vector $|0\rangle$ is contained in $\mathcal{H}_{\delta=0}$, which corresponds by (3.6) to the classical singularity $p=0$, defining the points in phase space where the two constraints (3.16) and (3.23) differ classically. Indeed, $|0\rangle$ is an eigenvector with eigenvalue 0 of (3.23)

$$
\hat{C}|0\rangle=\left(\frac{\sin \mu_{0} \hat{c}}{\mu_{0}}\right)^{2} \operatorname{sgn} \hat{\mathrm{p}} \widehat{\sqrt{|\mathrm{p}|}}|0\rangle=0,
$$

but not of (3.16). Hence the solutions of (3.23), given by (3.26), have a different structure than (3.25). There is no bounded solution with asymptotical behaviour $|\lambda|^{-\frac{1}{2}}$, instead there is the eigenvector $|0\rangle$. This eigenvector constitutes $\mathcal{H}_{0}^{p p}$ and has to be excluded from the following discussion, which deals with $\mathcal{H}_{0}^{a c}$ only (see section 2.3).

Although there is no analogue of (5.46) in the sector with $\delta=0$, the approximation that has been calculated gives

$$
\eta[|v\rangle] \approx \sum_{\lambda \in v+4 \mu_{0} \mathbb{Z}} \frac{1}{|\lambda+\nu|^{\frac{1}{4}}}\langle\lambda|,
$$

even for $v \in 4 \mu_{0} \mathbb{Z}$, i.e. in the sector $\delta=0$. So the approximation scheme treats all $\delta \in\left[0,4 \mu_{0}\right)$ equally. In the case for $\delta \neq 0,(5.44)$ has been interpreted as an approximation to the solution (5.46), which, as we have seen, does not exist in this sector. Thus, it is not clear which solution the rigging map approximates, if any. We attempt to interpret this as follows.

The disappearance of an analogue to the solution (5.46) for $\delta=0$ can be traced back to the appearance of sgn $\hat{\mathrm{p}} \widehat{\sqrt{|\mathrm{p}|}}$ in the constraint (3.23), which results in an eigenvector of $\hat{C}$ (hence $\hat{M}$ ) with eigenvalue 0 . The eigenvector $|0\rangle$ of $\hat{C}$ has to be removed from the analysis, since discrete and continuous spectra have to be treated separately in the direct integral decomposition ${ }^{4}$. Hence, the Hilbert space $\mathcal{H}_{0}$ has to be replaced by $\operatorname{span}\left\{|v\rangle \mid v \in 4 \mu_{0} \mathbb{Z} \backslash\{0\}\right\}$, which is a sub-Hilbert space in $\mathcal{H}_{0}$ of codimension 1 . The coherent states, however, are defined on all of $\mathcal{H}_{0}$; in particular the projection to $|0\rangle$ is nonzero for all coherent states in $\mathcal{H}_{0}$, i.e. states (3.31) with $\delta=0$. So, by using the coherent states for the semiclassical approximation on the sector $\delta=0$, one is extending the space one is allowed to look for solutions. So we have to conclude that the approximation provided by the coherent states is not appropriate for the sector $\delta=0$, since, strictly speaking, there are no coherent states living on this sector. Thus, the approximation cannot be trusted at all, and we conclude that there is no physical solution in the sector $\delta=0$. Hence, the whole physical Hilbert space is

$$
\mathcal{H}_{\text {phys }}=\mathcal{H}_{0}^{p p} \oplus \mathcal{H}_{0}^{a c}=\operatorname{span}\{|0\rangle\} \oplus \bigoplus_{\delta \in\left(0,4 \mu_{0}\right)} \mathbb{C} .=\bigoplus_{\delta \in\left[0,4 \mu_{0}\right)} \mathbb{C} .
$$

\footnotetext{
4 In particular, one has to perform the direct integral decomposition separately for each part of the spectrum, leading
} to a different choice of normalization vector $\left|\nu_{0}\right\rangle$ in (2.19) 


\subsection{Improvement of the model}

The constraint operator (3.23) was employed in [2], where it depended on the scale parameter $\mu_{0}$. A symmetrized version of this constraint was used in [4] to investigate quantum cosmology coupled to a massless scalar field. It was found that the fixed scale $\mu_{0}$ leads to serious problems with the low energy limit of the theory. Since $\mu_{0}$ serves as an area cutoff at small scales, it was then proposed in [5] to replace it by an operator expression depending on $\hat{p}$, which correctly implements the quantum behaviour of area at small scales.

One goes over to the eigenbasis $|v\rangle$ of the volume operator $\hat{V}$, which, since

$$
\hat{V} \sim \operatorname{sgn} \hat{p} \hat{p}^{\frac{3}{2}}
$$

is, of course, the same basis, just differently labelled. In this basis, the modified constraint operator (3.23) reads

$$
\hat{C}_{\text {mod }}=\sin \bar{\mu} \hat{c} \hat{A} \sin \bar{\mu} \hat{c}
$$

with

$$
\begin{aligned}
& \sin \bar{\mu} \hat{c}|v\rangle=\frac{|v+2\rangle-|v-2\rangle}{2} \\
& \hat{A}|v\rangle=K|v|[|v+1|-|v-1|]|v\rangle
\end{aligned}
$$

for some $K>0$. Thus, the operator acts as difference operator on the $|v\rangle$ with step size 1 , rather than with a step size depending on a parameter $\mu_{0}$. Writing this in a basis $|\mu\rangle$ of eigenvectors of $\hat{p}$, the action of this operator thus involves steps of $\mu$-dependent size.

Coupled to a massless matter field, this new constraint is able to deliver the correct low energy limit.

The same approximation technique we have employed in this paper could also have been applied to the modified constraint (5.51). But, since there are no matter fields present, the qualitative feature would have been the same, at least in the lower orders of our approximation. One can see this as follows: the master constraint we have employed acts as

$$
\hat{M}|\mu\rangle \sim \mu^{2} \sin ^{8} \mu_{0} \hat{c}|\mu\rangle
$$

for $|\mu| \gg 1$, on which the zero order of our approximation relied. The master constraint built from the modified constraint (5.51) acts as follows:

$$
\hat{M}_{\text {mod }}|v\rangle \sim v^{2} \sin ^{8} \bar{\mu} \hat{c}|v\rangle
$$

for $|v| \gg 1$. Note that (5.53) is the same as (5.52) with $\mu_{0}$ set to 1 . Of course, this is a completely different constraint now, since the labels $v$ and $\mu$ correspond to different physical entities, in particular volume and (densitized) area. But the mathematical behaviour is the same, if one forgets this. In particular, the approximation scheme will work exactly on similar lines with $\hat{M}_{\text {mod }}$ as with $\hat{M}$, and will deliver the same result.

This, of course, will change drastically, as soon as one incorporates matter into the model. The particular form of the matter constraints definitely depends on whether one works in the $|v\rangle$ - or the $|\mu\rangle$-basis. This is a nice illustration of the fact that one needs matter to distinguish certain physical quantities. It is worthwhile mentioning that this points into the direction of the concept of partial and complete observables, where the construction of Dirac observables for GR is built on relational connections between physical quantities [7, 15, 22].

\section{Summary and conclusion}

In this paper, we computed the physical inner product of a simple cosmological model. We did so in two different ways: firstly, we rewrote the classical side before quantization, in order 
to arrive at a much simpler constraint on the quantum side, the physical inner product of which could be computed easily. In each Hilbert space $\mathcal{H}_{\delta}$, there are a bounded and an unbounded solution to the constraint, and the bounded one was constituting the physical Hilbert space $\mathcal{H}_{\text {phys }}$, while the other solution was 'spurious', i.e. not contained in it. In the model calculated $H_{\text {phys }}$ is non-separable. This unphysical feature is a result of the quantization procedure, which breaks scale-invariance of the classical theory. Still, this was no particular problem for our calculations.

Secondly, we attempted to compute the physical inner product directly, without rewriting of the classical side. This resulted in a system which is classically equivalent up to points with $p=0$ on the phase space. The constraint of the new system is non-symmetric, which is why we employed the master constraint method to arrive at a self-adjoint constraint. Since the formulae for this operator were too complicated to compute exactly, we relied on the complexifier coherent states to approximate the physical inner product. This has led to interesting results.

Similarly to the case already observed, on each sector $\delta \in\left(0,4 \mu_{0}\right)$ there are a bounded and an unbounded solution, showing that rewriting on the classical side did not change the quantum theory significantly on the parts of the phase space where the two classical theories are equivalent. The sector $\delta=0$ contained the eigenvector of $\hat{p}$ belonging to vanishing momentum $p=0$, which defines the phase-space points where the two classical theories do not agree, and indeed the solution structure of the quantum constraints changed significantly. What did not change is the physical Hilbert space. Although now containing an eigenvector, $|0\rangle$, the resulting physical Hilbert space $\mathcal{H}_{\text {phys }}$ was isomorphic to the one computed from the simpler model. So rewriting the classical theory changed the structure of the solution space in quantum theory at the particular points where the classical theories disagree but, after going over to the physical Hilbert space, led to the right result.

The approximation of the physical inner product with the help of the complexifier coherent states was good enough to indicate that for each sector $\delta \in\left(0,4 \mu_{0}\right)$ the bounded solution was chosen to constitute the physical inner product, while all the unbounded solutions turned out to be spurious. This demonstrated first that going over to the master constraint did not change the quantum behaviour. In particular, although there are many more solutions to the master constraint $\hat{M}$ than to the original constraint $\hat{C}$, computing the physical inner product with $\hat{M}$ still resulted in a physical Hilbert space consisting of solutions to $\hat{C}$ only. Hence the additional solutions provided by the master constraint did not contribute to the physical Hilbert space, showing the strength of the master constraint programme.

This demonstrates that the complexifier coherent states provide a way to approximate physical inner products. Furthermore, the corrections to the approximated rigging map can in principle be calculated order by order (the 'perturbation parameter' in our case being the distance to the region of phase space where the approximation was to hold best) and be added to the previously obtained result, granting a way to improve the approximation by adding more and more correction terms.

\section{Acknowledgment}

BB would like to thank Christian Hillmann for pointing out the significance of formula (3.19).

\section{References}

[1] Abramowitz M and Stegun I E 1970 Handbook of Mathematical Functions (New York: Dover)

[2] Ashtekar A, Bojowald M and Lewandowski J 2003 Adv. Theor. Math. Phys. 7233 (Preprint gr-qc/0304074) 
[3] Ashtekar A, Lewandowski J, Marolf D, Mourão J and Thiemann T 1995 J. Math. Phys. 366456 (Preprint gr-qc/9504018)

[4] Ashtekar A, Pawlowski T and Singh P 2006 Phys. Rev. D 74124038 (Preprint gr-qc/0604013)

[5] Ashtekar A, Pawlowski T and Singh P 2006 Phys. Rev. D 74084003 (Preprint gr-qc/0607039)

[6] Dirac P A M 1964 Lectures in Quantum Mechanics (New York: Yeshive University)

[7] Dittrich B 2006 Class. Quantum Grav. 236155 (Preprint gr-qc/0507106)

[8] Dittrich B and Thiemann T 2006 Class. Quantum Grav. 231025 (Preprint gr-qc/0411138)

Dittrich B and Thiemann T 2006 Class. Quantum Grav. 231067 (Preprint gr-qc/0411139)

Dittrich B and Thiemann T 2006 Class. Quantum Grav. 231089 (Preprint gr-qc/0411140)

Dittrich B and Thiemann T 2006 Class. Quantum Grav. 231121 (Preprint gr-qc/0411141)

Dittrich B and Thiemann T 2006 Class. Quantum Grav. 231143 (Preprint gr-qc/0411142)

[9] Guilini D and Marolf D 1999 Class. Quantum Grav. 162489

[10] Hall B 1994 J. Funct. Anal. 122103

[11] Hall B 1997 J. Funct. Anal. 14398

[12] Hall B 1997 Commun. Math. Phys. 184233

[13] Henneaux M and Teitelboim C 1992 Quantization of Gauge Systems (Princeton, NJ: Princeton University Press)

[14] Noui K, Perez A and Vandersloot K 2005 Phys. Rev. D 71044025 (Preprint gr-qc/0411039)

[15] Rovelli C 2002 Phys. Rev. D 65124013 (Preprint gr-qc/0110035)

[16] Thiemann T 1998 Class. Quantum Grav. 15839 (Preprint gr-qc/9606089)

[17] Thiemann T 1998 Class. Quantum Grav. 15875 (Preprint gr-qc/9606090)

[18] Thiemann T 1998 Class. Quantum Grav. 151207 (Preprint gr-qc/9705017)

[19] Thiemann T 2006 Class. Quantum Grav. 232211 (Preprint gr-qc/0305080)

[20] Thiemann T 2006 Class. Quantum Grav. 232249 (Preprint gr-qc/0510011)

[21] Thiemann T 2001 Class. Quantum Grav. 182025 (Preprint hep-th/0005233)

[22] Thiemann T 2001 Introduction to modern canonical quantum general relativity Preprint gr-qc/0110034

[23] Thiemann T and Winkler O 2001 Class. Quantum Grav. 182561 (Preprint hep-th/0005237)

[24] Thiemann T and Winkler O 2001 Class. Quantum Grav. 184629 (Preprint hep-th/0005234)

[25] Thiemann T 2001 Class. Quantum Grav. 182025 (Preprint gr-qc/0206037) 